\title{
Dynamic Behavior Analysis of Intermediate Bearing-Squeeze Film Dampers-Rotor System under Constant Maneuvering Overload
}

\author{
Nan Zheng (iD, Mo-li Chen, Gui-Huo Luo iD, and Zhi-Feng Ye \\ Key Laboratory of Aero-Engine Thermal Environment and Structure, Ministry of Industry and Information Technology, \\ College of Energy and Power Engineering, Nanjing University of Aeronautics and Astronautics, Nanjing 210016, China
}

Correspondence should be addressed to Gui-Huo Luo; ghluo@nuaa.edu.cn

Received 13 January 2021; Accepted 24 April 2021; Published 3 May 2021

Academic Editor: Dario Richiedei

Copyright (c) 2021 Nan Zheng et al. This is an open access article distributed under the Creative Commons Attribution License, which permits unrestricted use, distribution, and reproduction in any medium, provided the original work is properly cited.

Under the flight maneuvering of an aircraft, the maneuvering load on the rotor is generated, which may induce the change of dynamic behavior of aeroengine rotor system. To study the influence on the rotor dynamic behavior of constant maneuvering overload, a nonlinear dynamic model of bearing-rotor system under arbitrary maneuver flight conditions is presented by finite element method. The numerical integral method is used to investigate the dynamic characteristics of the rotor model under constant maneuvering overload, and the simulation results are verified by experimental works. Based on this, the dynamic characteristics of a complex intermediate bearing-squeeze film dampers- (SFD-) rotor system during maneuvering flight are analyzed. The simulation results indicate that the subharmonic components are amplified under constant maneuvering overload. The amplitude of the combined frequency components induced by the coupling of the inner and outer rotors is weakened. The static displacements of the rotor caused by the additional excitation force are observed. Besides, the period stability of the movement of the rotor deteriorates during maneuver flight. The design of counterrotation of the inner and outer rotors can effectively reduce the amplitude of subharmonic under constant maneuvering overload.

\section{Introduction}

The maneuverability is an essential indicator in evaluating the performance of military aircraft. For military drones, it is unnecessary to consider the pilot's ability to withstand maneuvering loads, and the maneuvering overload will be significantly increased to improve the maneuverability in the future. Additional excitation load will be generated acting on the aeroengine rotor system under the condition of maneuver flight, which may affect the dynamic behavior of the rotor $[1,2]$. As the maneuvering overload increases, such effect may be magnified.

In recent years, significant attention has been paid to the dynamic behavior of the rotor system during maneuvering flight. $\mathrm{Xu}$ and Liao [3] studied the dynamic behavior of Jeffcott rotor with squeeze film dampers (SFD) under the condition of maneuver flight. The numerical simulation results manifested that the additional maneuvering load induced by maneuvering flight changes the vibration characteristics of the rotor system. The SFD can effectively suppress the transient vibration during the maneuvering process. Ananthan and Leishman [4] investigated the dynamic behavior of a turbo-shaft engine rotor system of a helicopter during maneuver flight. The numerical simulation results showed that the five types of maneuvering flight, that is, dive, jump, pulling up during descent, roll, and reverse roll, can affect the vibration amplitude of the rotor and generate unstable additional loads. Sakata et al. $[5,6]$ analyzed the steady-state vibration of a simply supported flexible rotor with rotary foundation through the methods of mathematical modeling, simulation calculation, and experimental methods.

Furthermore, Brentner et al. $[7,8]$ studied the noise problem of rotorcraft in maneuvering flight and developed a new system for maneuvering noise prediction. Yang et al. [9] studied the nonlinear dynamic response of a cracked rotor with rigid support during maneuvering flight, and three ways for the vibration responses leading to chaos were 
observed. Lin and Meng [10, 11] studied the vibration behavior of Jeffcott rotor under the conditions of initial bending, asymmetric stiffness, and rub-impact fault in the environment of maneuvering flight. Zhu and Chen [12, 13] established a dynamic model of an aircraft rotor system in arbitrary maneuvering flight action using Lagrange equations, in which the added damping, stiffness, and maneuvering load were obtained, and the influence of maneuver flight on the movement of rotor was investigated. Hou and Chen [14-16] established a dynamic model of a bearingrotor system under maneuver flight conditions with faults such as rub-impact and cracks. The nonlinear dynamics behavior of the system was analyzed through numerical integral and analytic methods. The subharmonic resonance and irregular motions caused by maneuver were observed. Gao et al. [17] established a flexible asymmetric rotor system with ball bearings and SFD during maneuver flight. The vibration characteristics in conditions of accelerative motion, angular motion, and pitching flight were studied.

Relative simple models, such as Jeffcott and lumped parameter rotor, are widely adopted in the above mentioned literature, which can efficiently disclose the nonlinear characteristics of rotor system. However, the analysis cannot precisely reflect the vibration behavior of the actual aeroengine complex rotor system. Using the finite element theory, the transient response of a rotor system with SFD during maneuver flight was investigated by Han and Ding [18], but a relative simple single rotor model was aimed in their research. Besides, there are few researches on the dynamic behavior of the rotor system during maneuver flight by experimental method.

This paper aims to establish a nonlinear dynamic model of bearing-rotor system under arbitrary maneuver flight conditions using FEM. The added damping, stiffness, and maneuvering load generated by maneuver flight are obtained. The numerical integral method is used to investigate the vibration behavior of the rotor under constant maneuvering overload, and the simulation results are verified by experiments. Based on this, the vibration characteristics of a complex intermediate bearing-SFD-rotor system under constant maneuvering overload are analyzed through the above methods.

\section{Mathematical Model}

\subsection{Finite Element Formulation}

2.1.1. Disk. To obtain the dynamic differential equations of a disk during maneuvering flight, a coordinate system is presented in Figure 1. OXYZ is the ground coordinate system, which represents the center of gravity, flight velocity, and acceleration of the plane. OXYZ is the coordinate system used to describe the whirl of the rotor around its own axis. Assuming that the engine rotor is installed on the aircraft with one end, which coincides with the center of gravity of the aircraft, then $O X Y Z$ is also the relative coordinate system of the aircraft. The degree-of-freedom of the disk can be expressed as

$$
q_{d}=\left[\begin{array}{llll}
x & y & \theta_{x} & \theta_{y}
\end{array}\right]
$$

And the kinetic energy of a disk of rotor system under arbitrary maneuvering flight actions can be written as

$$
T=T_{t}+T_{r}
$$

where $T_{t}$ and $T_{r}$ are the translational and rotational kinetic energy of the disk, respectively. And the translational kinetic energy during the movement of the rotor can be expressed as [13]

$$
T_{t}=\frac{1}{2} m\left(\dot{r}^{T} \dot{r}+v_{B}^{T} v_{B}-r^{T} \omega_{B}^{2} r+2 \dot{r}^{T} \omega_{B} r+2 v_{B}^{T} \dot{r}+2 v_{B}^{T} \omega_{B} r\right),
$$

where $m$ denotes the quality of the disk, $r$ represents the relative position of the disk in $O X Y Z$ coordinate system, and $v_{B}$ and $\omega_{B}$ denote the velocity and angular velocity of the aircraft in OXYZ, respectively. $r, v_{B}$, and $\omega_{B}$ can be expressed as follows:

$$
\begin{aligned}
& r=\left[\begin{array}{c}
x+e \cos (\Omega t+\varphi) \\
y+e \sin (\Omega t+\varphi) \\
z
\end{array}\right], \\
& v_{B}=\left[\begin{array}{c}
\dot{X}_{B} \\
\dot{Y}_{B} \\
\dot{Z}_{B}
\end{array}\right] \text {, } \\
& \omega_{B}=\left[\begin{array}{ccc}
0 & -\omega_{B, z} & \omega_{B, y} \\
\omega_{B, z} & 0 & -\omega_{B, x} \\
-\omega_{B, y} & \omega_{B, x} & 0
\end{array}\right] \text {, }
\end{aligned}
$$

where $\Omega$ denotes the rotating speed of the rotor, $\varphi$ is the initial phase angle, and $\dot{X}_{B}, \dot{Y}_{B}, \dot{Z}_{B}$ and $\omega_{B, x}, \omega_{B, y}, \omega_{B, z}$ are the velocities of the aircraft along each coordinate axis and the angular velocities around each coordinate axis in $O X Y Z$ coordinate system, respectively.

The rotational kinetic energy of the disk during the movement of the rotor is [13]

$$
T_{r}=\frac{1}{2} I_{d}\left(\dot{\theta}_{x}+\omega_{B, x}\right)^{2}+\frac{1}{2} I_{d}\left(\dot{\theta}_{y}+\omega_{B, y}\right)^{2}+\frac{1}{2} I_{p}\left[\Omega^{2}+2 \Omega\left(\theta_{y}+\theta_{B, y}\right)\left(\dot{\theta}_{x}+\omega_{B, x}\right)\right],
$$

where $\theta_{B, y}$ denotes the angular displacement of the aircraft around $Y$ axis in OXYZ coordinate system, and $\theta_{y}+\theta_{B, y} \approx \theta_{y}, \quad\left(\theta_{y}+\theta_{B, y}\right)^{\prime} \approx \dot{\theta}_{y}+\omega_{B, y}, I_{d}$ and $I_{p}$ are the diameter and polar moment of inertia of the disk, respectively. Considering the damping of the disk as a linear function of velocity, and assuming that the generalized 


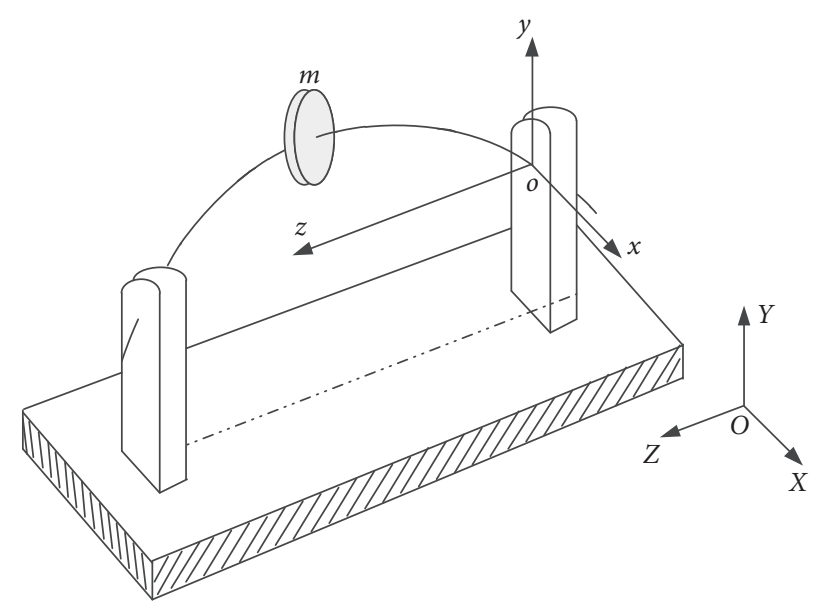

Figure 1: Coordinate system of disk.

damping matrix of the disk is $C_{d}$, the generalized stiffness matrix of the shaft at the position of the disk is $K_{d}$. Then, the dissipation energy of the disk can be expressed as equation (6) by the Rayleigh energy dissipation function. And the elastic potential energy of the flexible shaft at the position of the disk can be expressed as

$$
\begin{aligned}
& D=\frac{1}{2} \dot{q}_{d}^{T} C_{d} \dot{q}_{d}, \\
& V=\frac{1}{2} q_{d}^{T} K_{d} q_{d} .
\end{aligned}
$$

Substitute equations (1)-(6) into the Lagrange equations for nonconservative systems, and the dynamic differential equations of the disk during arbitrary maneuver flight actions can be deduced as

$$
\begin{aligned}
& M_{d} \ddot{q}_{d}+\left(C_{d}+\Omega G_{d}+C_{d b}\right) \dot{q}_{d}+\left(K_{d}+K_{d b}\right) q_{d}=F_{u}+F_{d b}+Q_{d}, \\
& C_{d b}=\left[\begin{array}{cccc}
0 & -2 m \omega_{B, z} & 0 & 0 \\
2 m \omega_{B, z} & 0 & 0 & 0 \\
0 & 0 & 0 & 0 \\
0 & 0 & 0 & 0
\end{array}\right] \\
& K_{d b}=\left[\begin{array}{cccc}
-m\left(\omega_{B, y}^{2}+\omega_{B, z}^{2}\right) & m\left(\omega_{B, x} \omega_{B, y}-\dot{\omega}_{B, z}\right) & 0 & 0 \\
m\left(\omega_{B, x} \omega_{B, y}+\dot{\omega}_{B, z}\right) & -m\left(\omega_{B, x}^{2}+\omega_{B, z}^{2}\right) & 0 & 0 \\
0 & 0 & 0 & 0 \\
0 & 0 & 0 & 0
\end{array}\right] \text {, } \\
& F_{d b}=\left[\begin{array}{c}
-m\left(\ddot{X}_{B}-\omega_{B, z} \dot{Y}_{B}+\omega_{B, y} \dot{Z}_{B}\right)-m z\left(\omega_{B, x} \omega_{B, z}+\dot{\omega}_{B, y}\right) \\
-m\left(\ddot{Y}_{B}+\omega_{B, z} \dot{X}_{B}-\omega_{B, x} \dot{Z}_{B}\right)-m z\left(\omega_{B, y} \omega_{B, z}-\dot{\omega}_{B, x}\right) \\
-I_{p} \Omega \omega_{B, y}-I_{d} \dot{\omega}_{B, x} \\
I_{p} \Omega \omega_{B, x}-I_{d} \dot{\omega}_{B, y}
\end{array}\right],
\end{aligned}
$$

where $M_{d}$ and $G_{d}$ are the inertial matrix and gyro matrix of the disk with maneuver flight ignored, respectively. Their formulas are omitted in this paper, which can be found in Ref. [19]. $C_{d b}, K_{d b}$, and $F_{d b}$ denote the added damping matrix, stiffness matrix, and maneuvering load caused by maneuver flight. $F_{u}$ represents the unbalanced force of the disk, and $Q_{d}$ denotes the generalized external forces acting on the disk. 


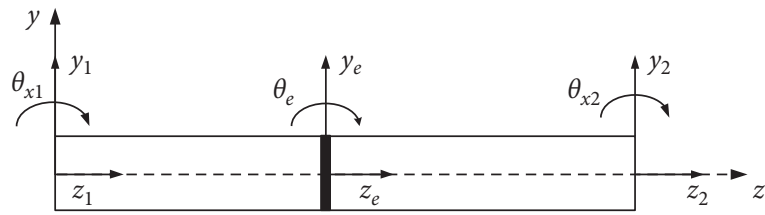

(a)

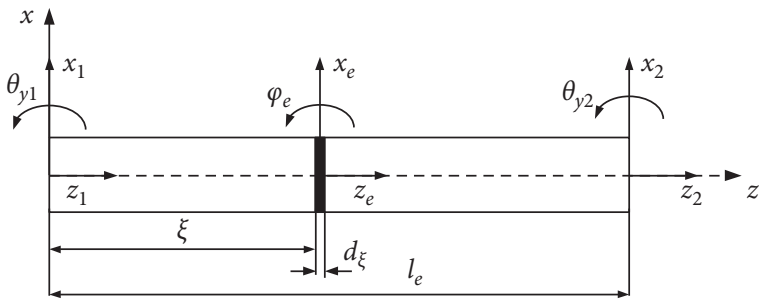

(b)

FIgURE 2: Schematic diagram of beam element.

2.1.2. Shaft. The schematic diagram of a Timoshenko beam element is shown in Figure 2. Similar to the disk, the kinetic energy of an arbitrary microelement segment of length $d_{\xi}$ can be expressed as follows:

$$
\begin{aligned}
\mathrm{d} T= & \mathrm{d} T_{t}+\mathrm{d} T_{r}=\frac{1}{2} \rho_{e} A_{e}\left(\dot{r}_{e}^{T} \dot{r}_{e}+v_{B}^{T} v_{B}-r_{e}^{T} \omega_{B}^{2} r_{e}+2 \dot{r}_{e}^{T} \omega_{B} r_{e}+2 v_{B}^{T} \dot{r}_{e}+2 v_{B}^{T} \omega_{B} r_{e}\right) \mathrm{d} \xi \\
& +\frac{1}{2} \mathrm{~d} I_{d}\left(\dot{\theta}_{e}+\omega_{B, x}\right)^{2}+\frac{1}{2} \mathrm{~d} I_{d}\left(\dot{\varphi}_{e}+\omega_{B, y}\right)^{2}+\frac{1}{2} \mathrm{~d} I_{p}\left[\Omega^{2}+2 \Omega\left(\dot{\theta}_{e}+\omega_{B, x}\right)\left(\varphi_{e}+\theta_{B, y}\right)\right], \\
r_{e}= & {\left[\begin{array}{c}
x_{e} \\
y_{e} \\
z_{e}
\end{array}\right], } \\
{\left[\begin{array}{c}
x_{e} \\
y_{e} \\
\theta_{e} \\
\varphi_{e}
\end{array}\right]=} & {\left[\begin{array}{cccccccc}
N_{1} & 0 & 0 & N_{2} & N_{3} & 0 & 0 & N_{4} \\
0 & N_{1} & -N_{2} & 0 & 0 & N_{3} & -N_{4} & 0 \\
0 & -\breve{N}_{1} & \breve{N}_{2} & 0 & 0 & -\breve{N}_{3} & \breve{N}_{4} & 0 \\
\breve{N}_{1} & 0 & 0 & \breve{N}_{2} & \breve{N}_{3} & 0 & 0 & \check{N}_{4}
\end{array}\right] q_{e}, } \\
q_{e}= & {\left[\begin{array}{lllllllll}
x_{1} & y_{1} & \theta_{x 1} & \theta_{y 1} & x_{2} & y_{2} & \theta_{x 2} & \theta_{y 2}
\end{array}\right]^{T}, }
\end{aligned}
$$

where $q_{e}$ is the nodal displacement vector, and $N_{i}(i=1-4)$ and $N_{i}(i=1-4)$ represent the shape functions and the expressions, which are referred to in Ref. [19]. $r_{e}$ denotes the relative position of the centroid of the microelement segment in $o x y z$ coordinate system. Then, the kinetic energy expression of the beam element can be obtained by integrating equation (8) along the axis $o z$.

Similar to the derivation process of a disk, the dynamic differential equations of the beam element under arbitrary maneuver flight conditions can be expressed as

$$
M_{e} \ddot{q}_{e}+\left(C_{e}+\Omega G_{e}+C_{e b}\right) \dot{q}_{e}+\left(K_{e}+K_{e b}\right) q_{e}=F_{e b}+Q_{e},
$$

where $M_{e}, G_{e}$, and $K_{e}$ are the inertial matrix, gyro matrix, and stiffness matrix of the beam element, and the expressions are referred to in Ref. [19]. In this work, the internal damping of the material is ignored, and $C_{e}$ is the external damping of the beam element. $Q_{e}$ denotes the generalized external forces applied to the beam element. $C_{e b}, K_{e b}$, and $F_{e b}$ denote the added damping, stiffness, and maneuvering load caused by maneuver flight, which can be expressed as follows:

$$
\begin{aligned}
C_{e b}= & 2 \rho_{e} A_{e} \omega_{B, z} H_{1}, \\
K_{e b}= & \rho_{e} A_{e}\left(\omega_{B, y}^{2}+\omega_{B, z}^{2}\right) H_{2}+\rho_{e} A_{e}\left(\omega_{B, x}^{2}+\omega_{B, z}^{2}\right) H_{3}+\rho_{e} A_{e}\left(\omega_{B, x} \omega_{B, y}-\dot{\omega}_{B, z}\right) H_{4} \\
& +\rho_{e} A_{e}\left(\omega_{B, x} \omega_{B, y}+\dot{\omega}_{B, z}\right) H_{5},
\end{aligned}
$$


$F_{e b}=\rho_{e} A_{e}\left[\begin{array}{c}\left(\dot{Y}_{B} \omega_{B, z}-\dot{Z}_{B} \omega_{B, y}-\dot{\omega}_{B, y} z_{e}-\ddot{X}_{B}-\omega_{B, x} \omega_{B, z} z_{e}\right) A_{1} \\ \left(-\dot{X}_{B} \omega_{B, z}+\dot{Z}_{B} \omega_{B, x}+\dot{\omega}_{B, x} z_{e}-\ddot{Y}_{B}-\omega_{B, y} \omega_{B, z} z_{e}\right) A_{1} \\ \left(\dot{X}_{B} \omega_{B, z}-\dot{Z}_{B} \omega_{B, x}-\dot{\omega}_{B, x} z_{e}+\ddot{Y}_{B}+\omega_{B, y} \omega_{B, z} z_{e}\right) A_{2} \\ \left(\dot{Y}_{B} \omega_{B, z}-\dot{Z}_{B} \omega_{B, y}-\dot{\omega}_{B, y} z_{e}-\ddot{X}_{B}-\omega_{B, x} \omega_{B, z} z_{e}\right) A_{2} \\ \left(\dot{Y}_{B} \omega_{B, z}-\dot{Z}_{B} \omega_{B, y}-\dot{\omega}_{B, y} z_{e}-\ddot{X}_{B}-\omega_{B, x} \omega_{B, z} z_{e}\right) A_{3} \\ \left(-\dot{X}_{B} \omega_{B, z}+\dot{Z}_{B} \omega_{B, x}+\dot{\omega}_{B, x} z_{e}-\ddot{Y}_{B}-\omega_{B, y} \omega_{B, z} z_{e}\right) A_{3} \\ \left(\dot{X}_{B} \omega_{B, z}-\dot{Z}_{B} \omega_{B, x}-\dot{\omega}_{B, x} z_{e}+\ddot{Y}_{B}+\omega_{B, y} \omega_{B, z} z_{e}\right) A_{4} \\ \left(\dot{Y}_{B} \omega_{B, z}-\dot{Z}_{B} \omega_{B, y}-\dot{\omega}_{B, y} z_{e}-\ddot{X}_{B}-\omega_{B, x} \omega_{B, z} z_{e}\right) A_{4}\end{array}\right]+\left[\begin{array}{c}\left(2 \Omega \omega_{B, x}-\dot{\omega}_{B, y}\right) \\ \left(2 \Omega \omega_{B, y}+\dot{\omega}_{B, x}\right) B_{1} \\ -\left(2 \Omega \omega_{B, y}+\dot{\omega}_{B, x}\right) B_{2} \\ \left(2 \Omega \omega_{B, x}-\dot{\omega}_{B, y}\right) B_{2} \\ \left(2 \Omega \omega_{B, x}-\dot{\omega}_{B, y}\right) B_{3} \\ \left(2 \Omega \omega_{B, y}+\dot{\omega}_{B, x}\right) B_{3} \\ -\left(2 \Omega \omega_{B, y}+\dot{\omega}_{B, x}\right) B_{4} \\ \left(2 \Omega \omega_{B, x}-\dot{\omega}_{B, y}\right) B_{4}\end{array}\right]$,

$H_{1}=\left[\begin{array}{cccccccc}0 & A_{11} & A_{12} & 0 & 0 & -A_{13} & A_{14} & 0 \\ A_{11} & 0 & 0 & A_{12} & A_{13} & 0 & 0 & A_{14} \\ -A_{12} & 0 & 0 & -A_{22} & -A_{23} & 0 & 0 & -A_{24} \\ 0 & -A_{12} & A_{22} & 0 & 0 & -A_{23} & A_{24} & 0 \\ 0 & -A_{13} & A_{23} & 0 & 0 & -A_{33} & A_{34} & 0 \\ A_{13} & 0 & 0 & A_{23} & A_{33} & 0 & 0 & A_{34} \\ -A_{14} & 0 & 0 & -A_{24} & -A_{34} & 0 & 0 & -A_{44} \\ 0 & -A_{14} & A_{24} & 0 & 0 & -A_{34} & A_{44} & 0\end{array}\right]$,

$H_{2}=\left[\begin{array}{cccccccc}-A_{11} & 0 & 0 & -A_{12} & -A_{13} & 0 & 0 & -A_{14} \\ 0 & 0 & 0 & 0 & 0 & 0 & 0 & 0 \\ 0 & 0 & 0 & 0 & 0 & 0 & 0 & 0 \\ -A_{12} & 0 & 0 & -A_{22} & -A_{23} & 0 & 0 & -A_{24} \\ -A_{13} & 0 & 0 & -A_{23} & -A_{33} & 0 & 0 & -A_{34} \\ 0 & 0 & 0 & 0 & 0 & 0 & 0 & 0 \\ 0 & 0 & 0 & 0 & 0 & 0 & 0 & 0 \\ -A_{14} & 0 & 0 & -A_{24} & -A_{34} & 0 & 0 & -A_{44}\end{array}\right]$,

$H_{3}=\left[\begin{array}{cccccccc}0 & 0 & 0 & 0 & 0 & 0 & 0 & 0 \\ 0 & -A_{11} & A_{12} & 0 & 0 & -A_{13} & A_{14} & 0 \\ 0 & A_{12} & -A_{22} & 0 & 0 & A_{23} & -A_{24} & 0 \\ 0 & 0 & 0 & 0 & 0 & 0 & 0 & 0 \\ 0 & 0 & 0 & 0 & 0 & 0 & 0 & 0 \\ 0 & -A_{13} & A_{23} & 0 & 0 & -A_{33} & A_{34} & 0 \\ 0 & A_{14} & -A_{24} & 0 & 0 & A_{34} & -A_{44} & 0 \\ 0 & 0 & 0 & 0 & 0 & 0 & 0 & 0\end{array}\right]$,

$H_{4}=\left[\begin{array}{cccccccc}0 & A_{11} & -A_{12} & 0 & 0 & A_{13} & -A_{14} & 0 \\ 0 & 0 & 0 & 0 & 0 & 0 & 0 & 0 \\ 0 & 0 & 0 & 0 & 0 & 0 & 0 & 0 \\ 0 & A_{12} & -A_{22} & 0 & 0 & A_{23} & -A_{24} & 0 \\ 0 & A_{13} & -A_{23} & 0 & 0 & A_{33} & -A_{34} & 0 \\ 0 & 0 & 0 & 0 & 0 & 0 & 0 & 0 \\ 0 & 0 & 0 & 0 & 0 & 0 & 0 & 0 \\ 0 & A_{14} & -A_{24} & 0 & 0 & A_{34} & -A_{44} & 0\end{array}\right]$, 


$$
H_{5}=\left[\begin{array}{cccccccc}
0 & 0 & 0 & 0 & 0 & 0 & 0 & 0 \\
A_{11} & 0 & 0 & A_{12} & A_{13} & 0 & 0 & A_{14} \\
-A_{12} & 0 & 0 & -A_{22} & -A_{23} & 0 & 0 & -A_{24} \\
0 & 0 & 0 & 0 & 0 & 0 & 0 & 0 \\
0 & 0 & 0 & 0 & 0 & 0 & 0 & 0 \\
A_{13} & 0 & 0 & A_{23} & A_{33} & 0 & 0 & A_{34} \\
-A_{14} & 0 & 0 & -A_{24} & -A_{34} & 0 & 0 & -A_{44} \\
0 & 0 & 0 & 0 & 0 & 0 & 0 & 0
\end{array}\right],
$$

where

$$
\left\{\begin{array}{rl}
A_{i} & =\int_{0}^{l_{e}} N_{i} \mathrm{~d} \xi \\
B_{i} & =\int_{0}^{l_{e}} \breve{N}_{i} \mathrm{~d} \xi \\
A_{i j} & =\int_{0}^{l_{e}} N_{i} N_{j} \mathrm{~d} \xi, \\
B_{i j} & =\int_{0}^{l_{e}} \breve{N}_{i} \breve{N}_{j} \mathrm{~d} \xi,
\end{array} \quad(i=1 \sim 4, j=1 \sim 4),\right.
$$

in which $\rho_{e}, A_{e}$, and $I_{e}$ denote the material density, the crosssectional area, and the area moment of the shaft inertia, respectively.

Rearranging the differential equations of motion of the disks and beam elements, the dynamic differential equations of the rotor system under arbitrary maneuver flight actions can be expressed as

$$
M \ddot{q}+\left(C+\Omega G+C_{b}\right) \dot{q}+\left(K+K_{b}\right) q=F_{u}+F_{b}+Q
$$

where $M, C, G$, and $K$ represent the inertial matrix, damping matrix, gyro moment matrix, and stiffness matrix of the rotor system without maneuver flight conditions and bending-torsion coupling effects considered, respectively. $C_{b}, K_{b}$, and $F_{b}$ are the added damping matrix, stiffness matrix, and maneuvering load vectors caused by maneuver flight. $Q$ denotes the generalized external force, which is applied to the rotor system containing the bearing force at the support stage.

2.2. Nonlinear Forces of the Bearing. In the process of the whirling of the rotor, the nonlinear forces of rolling ball bearing can be written as follows [20]:

$$
\left\{\begin{array}{l}
f_{x}^{b}=-k_{n} \sum_{j=1}^{N_{b}} u_{\theta j}^{\xi} H\left(u_{\theta j}\right) \cos \theta_{j}, \\
f_{y}^{b}=-k_{n} \sum_{j=1}^{N_{b}} u_{\theta j}^{\xi} H\left(u_{\theta j}\right) \sin \theta_{j}, \\
\theta_{j}=\frac{2 \pi(j-1)}{N_{b}}+\omega_{b m} t
\end{array}\right.
$$

$$
\begin{gathered}
u_{\theta j}=x \cos \theta_{j}+y \sin \theta_{j}-\frac{\gamma}{2}, \\
H\left(u_{\theta j}\right) \begin{cases}0, & u_{\theta j} \leq 0, \\
u_{\theta j}, & u_{\theta j}>0,\end{cases} \\
\omega_{b m}=\frac{\omega_{\text {in }} r_{1}+\omega_{\text {out }} r_{2}}{r_{1}+r_{2}},
\end{gathered}
$$

where $k_{n}$ denotes the contact stiffness between the roller and the ring. $N_{b}$ is the number of rollers. $\theta_{j}$ denotes the circumferential position of the $j$-th roller at time $t$. And $\gamma$ is the radial clearance. $u_{\theta j}$ is the elastic contact deformation in the radial direction of the $j$-th roller. Furthermore, $\omega_{b m}$ denotes the rotational speed of the revolution of rollers. $r_{1}$ and $r_{2}$ are the internal and external radius of the bearing. $\omega_{\text {in }}$ and $\omega_{\text {out }}$ represent the rotational speeds of the internal and external rings. Generally, $\xi=(3 / 2)$ is set for rolling ball bearing.

Substituting the nonlinear forces of bearing into the generalized external force $Q$ in equation (12), the nonlinear dynamic model of the bearing-rotor system can be obtained.

\section{Experimental Verification for the Bearing- Rotor System}

3.1. Test Rig Description. To validate the correctness of the dynamic model presented in this paper, a test rig for simulating the maneuvering flight of a bearing-rotor system is proposed and designed, as shown in Figure 3. The rotor system is fixed at one end of the rotating arm structure using an installing seat. The other end of the rotating arm is mounted with balancing weights. During the experiments, the rotating arm structure is driven by the driving motor through the reduction gear box. It leads to the bearing-rotor system to rotate around the central spindle of the test rig. Through the different mounting holes reserved on the installing seat, the rotor can provide different pitch angles, as shown in Figure 4 . When the pitch angle is set to $0^{\circ}$, the maneuver flight action of hovering around the central spindle is simulated. When the pitch angle is $90^{\circ}$, the maneuver flight action of rolling is performed. And if the pitch angle is between $0^{\circ}$ and $90^{\circ}$, the synthetic maneuver of hovering and rolling can be studied. The test rig can apply the additional centrifugal force and gyro moment to the rotor system caused by maneuver flight. The maneuvering 


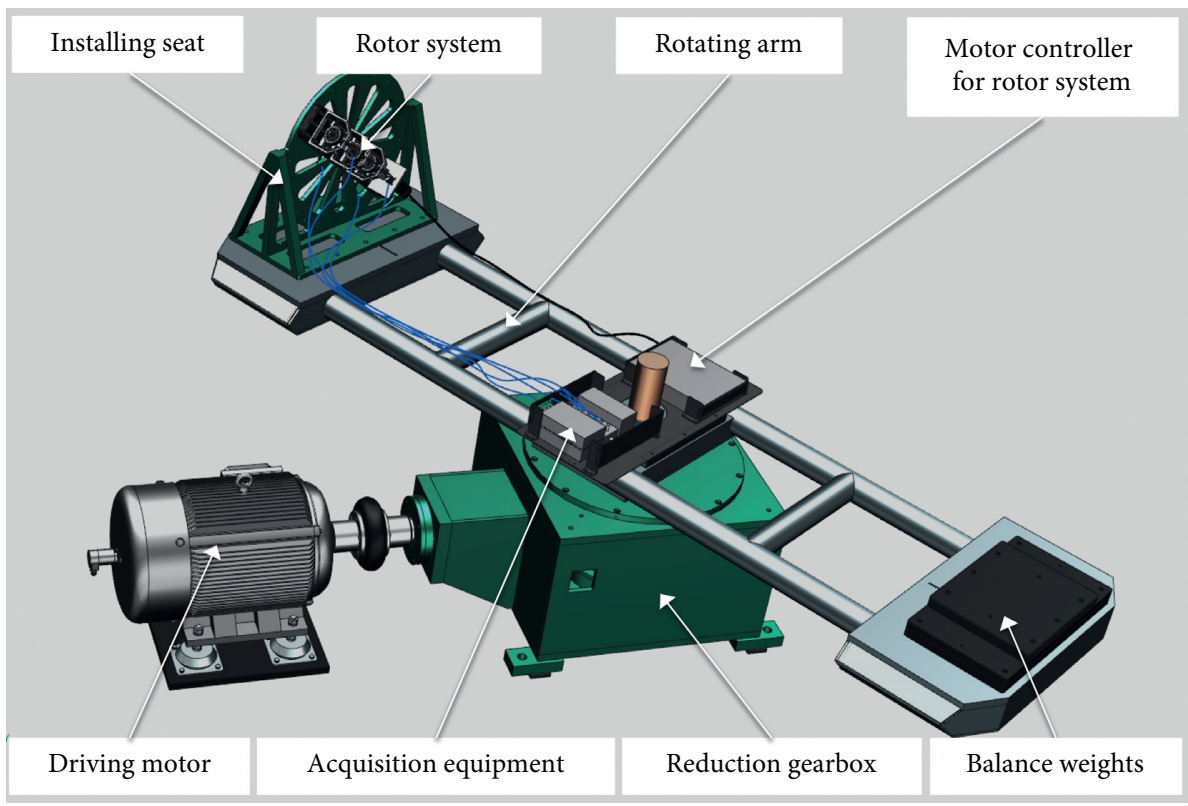

FIgURE 3: Test rig for simulating maneuver flight of a bearing-rotor system.

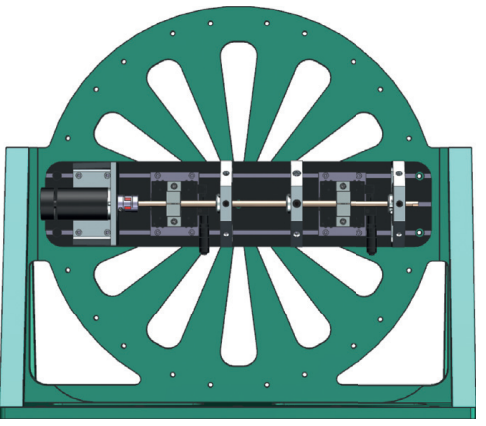

(a)

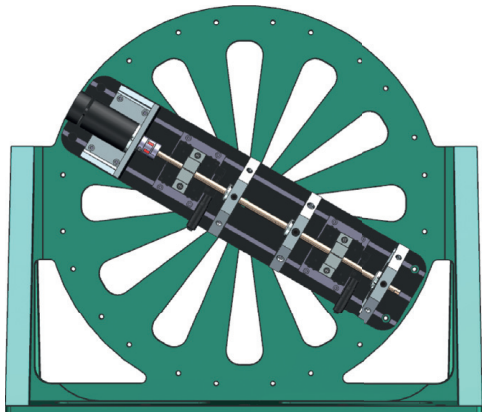

(b)

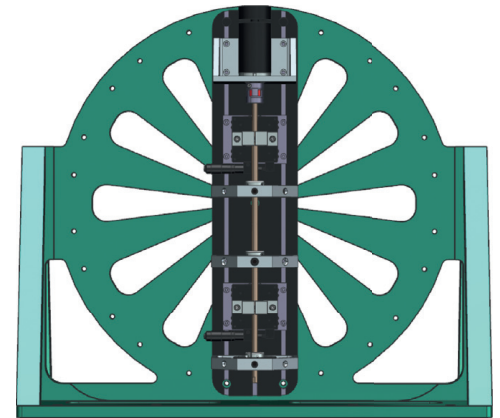

(c)

Figure 4: Diagrams of pitch angle. (a) $0^{\circ}$. (b) $30^{\circ}$. (c) $90^{\circ}$.

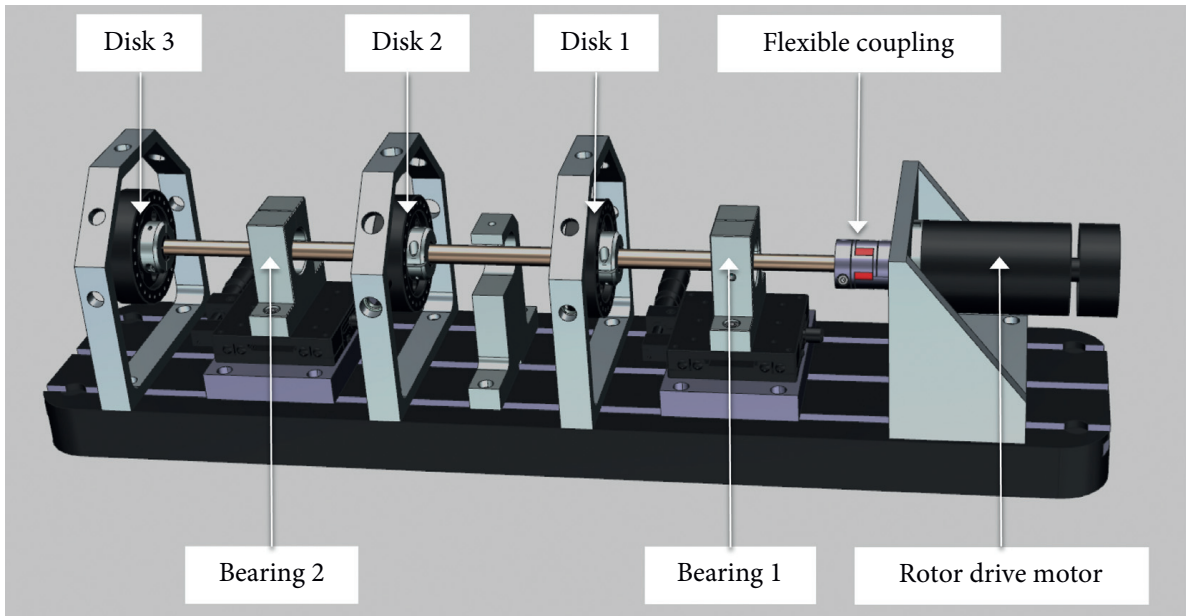

FIGURE 5: Bearing-rotor system. 
TABLE 1: Inertial properties and unbalance configuration.

\begin{tabular}{lccc}
\hline Name & Disk 1 & Disk 2 & Disk 3 \\
\hline Mass $(\mathrm{kg})$ & 0.4 & 0.4 & 0.5 \\
Polar moment of inertia $\left(\mathrm{g} \cdot \mathrm{m}^{2}\right)$ & 0.00038 & 0.00038 & 0.00041 \\
Unbalance $\left(10^{-5} \mathrm{~kg} \cdot \mathrm{m}\right)$ & 4 & 4 & 5 \\
\hline
\end{tabular}

TABLE 2: Parameters of the bearings.

\begin{tabular}{lcc}
\hline Name & Bearing 1 & Bearing 2 \\
\hline Radius of outer ring $(\mathrm{mm})$ & 30 & 30 \\
Radius of inner ring $(\mathrm{mm})$ & 10 & 10 \\
No. of rollers & 8 & 8 \\
Radial clearance $(\mu \mathrm{m})$ & 6 & 6 \\
\hline
\end{tabular}

TABle 3: Maneuver flight parameters.

\begin{tabular}{|c|c|c|c|c|c|c|}
\hline & Flight maneuvers & Overload (g) & $\dot{Z}_{B}(\mathrm{~m} / \mathrm{s})$ & $\omega_{B, y}(\mathrm{rad} / \mathrm{s})$ & $\dot{Y}_{B}(\mathrm{~m} / \mathrm{s})$ & $\omega_{B, z}(\mathrm{rad} / \mathrm{s})$ \\
\hline 1 & Hovering & 10 & 12.12 & 8.08 & 0 & 0 \\
\hline 2 & Hovering & 20 & 17.15 & 11.43 & 0 & 0 \\
\hline 3 & Hovering & 30 & 21 & 14 & 0 & 0 \\
\hline 4 & Rolling & 10 & 0 & 0 & 12.12 & 8.08 \\
\hline 5 & Rolling & 20 & 0 & 0 & 17.15 & 11.43 \\
\hline 6 & Rolling & 30 & 0 & 0 & 21 & 14 \\
\hline 7 & Synthetic & 10 & 10.5 & 7 & 6.06 & 4.03 \\
\hline 8 & Synthetic & 20 & 14.85 & 9.90 & 8.57 & 5.72 \\
\hline 9 & Synthetic & 30 & 18.19 & 12.12 & 10.5 & 7 \\
\hline
\end{tabular}

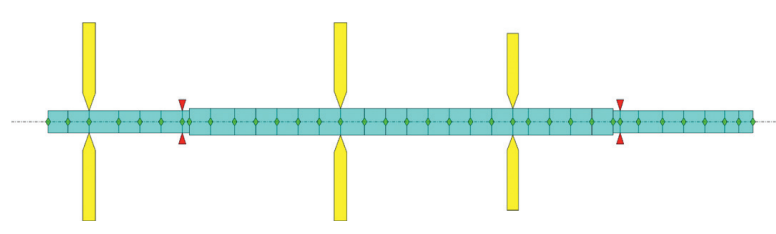

Figure 6: The mesh diagram of the bearing-rotor system.

TABLe 4: Dimension and elements information of the bearing-rotor system.

\begin{tabular}{|c|c|c|c|c|c|}
\hline Node number & Axial location $(\mathrm{m})$ & Bearing/disk & Element number & Outer diameter $(\mathrm{m})$ & Inner diameter $(\mathrm{m})$ \\
\hline 1 & 0.001 & \multirow{6}{*}{ Disk 3} & 1 & 0.010 & 0 \\
\hline 2 & 0.015 & & 2 & 0.010 & 0 \\
\hline 3 & 0.030 & & 3 & 0.010 & 0 \\
\hline 4 & 0.051 & & 4 & 0.010 & 0 \\
\hline 5 & 0.066 & & 5 & 0.010 & 0 \\
\hline 6 & 0.081 & & 6 & 0.010 & 0 \\
\hline 7 & 0.096 & \multirow{8}{*}{ Bearing 2} & 7 & 0.010 & 0 \\
\hline 8 & 0.101 & & 8 & 0.012 & 0 \\
\hline 9 & 0.116 & & 9 & 0.012 & 0 \\
\hline 10 & 0.133 & & 10 & 0.012 & 0 \\
\hline 11 & 0.148 & & 11 & 0.012 & 0 \\
\hline 12 & 0.163 & & 12 & 0.012 & 0 \\
\hline 13 & 0.178 & & 13 & 0.012 & 0 \\
\hline 14 & 0.193 & & 14 & 0.012 & 0 \\
\hline
\end{tabular}


TABle 4: Continued.

\begin{tabular}{|c|c|c|c|c|c|}
\hline Node number & Axial location $(\mathrm{m})$ & Bearing/disk & Element number & Outer diameter $(\mathrm{m})$ & Inner diameter $(\mathrm{m})$ \\
\hline 15 & 0.208 & \multirow{8}{*}{ Disk 2} & 15 & 0.012 & 0 \\
\hline 16 & 0.225 & & 16 & 0.012 & 0 \\
\hline 17 & 0.240 & & 17 & 0.012 & 0 \\
\hline 18 & 0.255 & & 18 & 0.012 & 0 \\
\hline 19 & 0.270 & & 19 & 0.012 & 0 \\
\hline 20 & 0.285 & & 20 & 0.012 & 0 \\
\hline 21 & 0.300 & & 21 & 0.012 & 0 \\
\hline 22 & 0.315 & & 22 & 0.012 & 0 \\
\hline 23 & 0.330 & \multirow{6}{*}{ Disk 1} & 23 & 0.012 & 0 \\
\hline 24 & 0.341 & & 24 & 0.012 & 0 \\
\hline 25 & 0.356 & & 25 & 0.012 & 0 \\
\hline 26 & 0.371 & & 26 & 0.012 & 0 \\
\hline 27 & 0.386 & & 27 & 0.012 & 0 \\
\hline 28 & 0.401 & & 28 & 0.010 & 0 \\
\hline 29 & 0.406 & \multirow{8}{*}{ Bearing 1} & 29 & 0.010 & 0 \\
\hline 30 & 0.419 & & 30 & 0.010 & 0 \\
\hline 31 & 0.436 & & 31 & 0.010 & 0 \\
\hline 32 & 0.451 & & 32 & 0.010 & 0 \\
\hline 33 & 0.466 & & 33 & 0.010 & 0 \\
\hline 34 & 0.480 & & 34 & 0.010 & 0 \\
\hline 35 & 0.490 & & 35 & 0.010 & 0 \\
\hline 36 & 0.500 & & & & \\
\hline
\end{tabular}

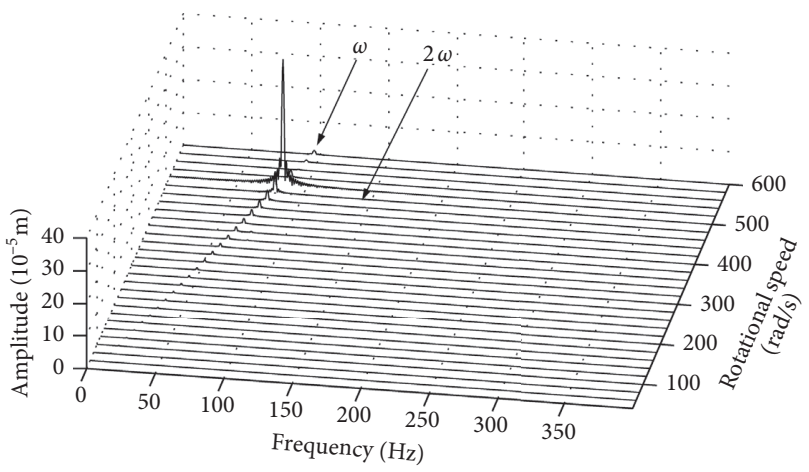

(a)

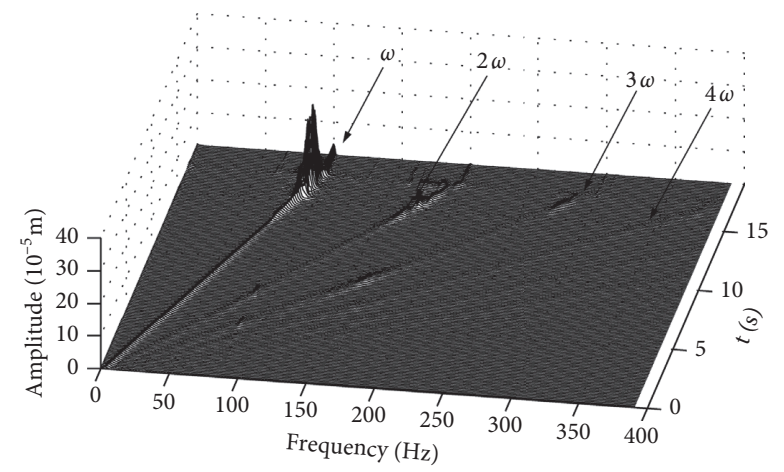

(b)

FIGURE 7: Waterfall spectra plots for the vibration of disk 3 without maneuver flight. (a) Numerical simulation. (b) Experimental results.

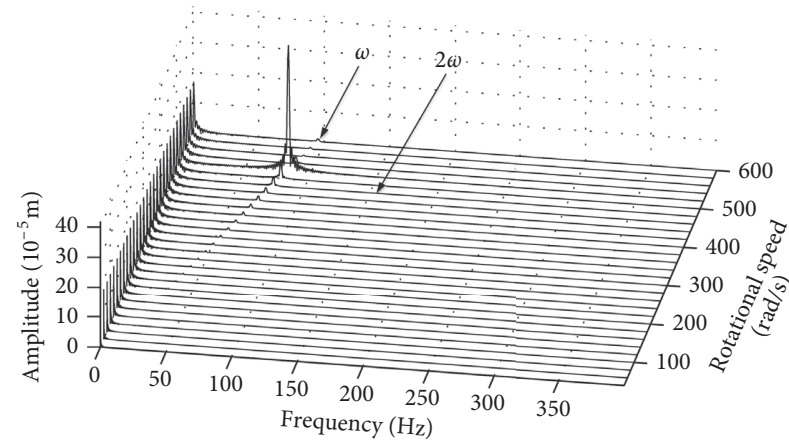

(a)

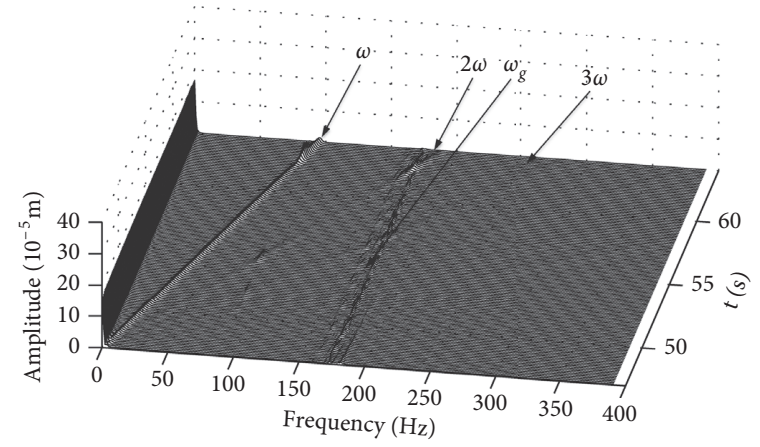

(b)

FIgURE 8: Continued. 


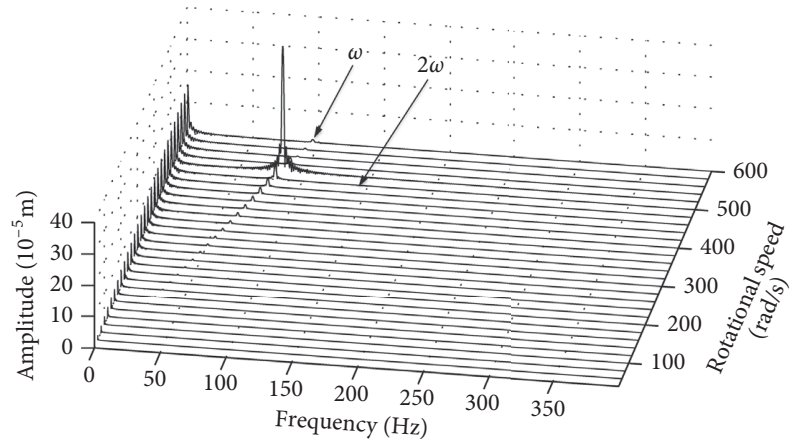

(c)

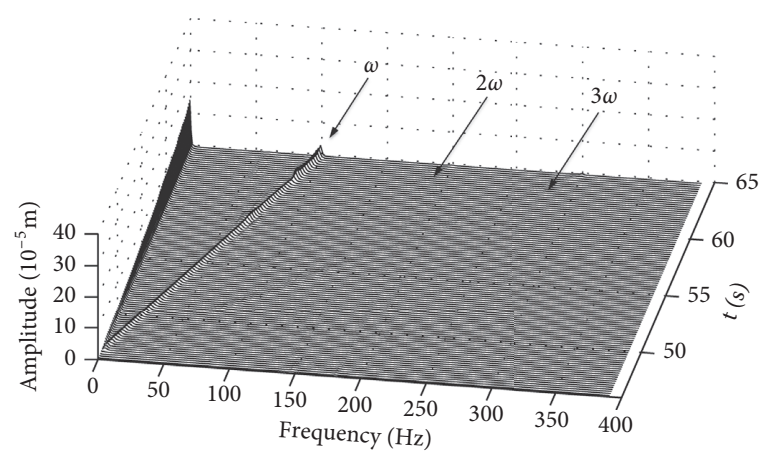

(d)

FIgURE 8: Waterfall spectra plots for the vibration of disk 3 during hovering. (a) Horizontal response, numerical simulation. (b) Horizontal response, experimental results. (c) Vertical response, numerical simulation. (d) Vertical response, experimental results.

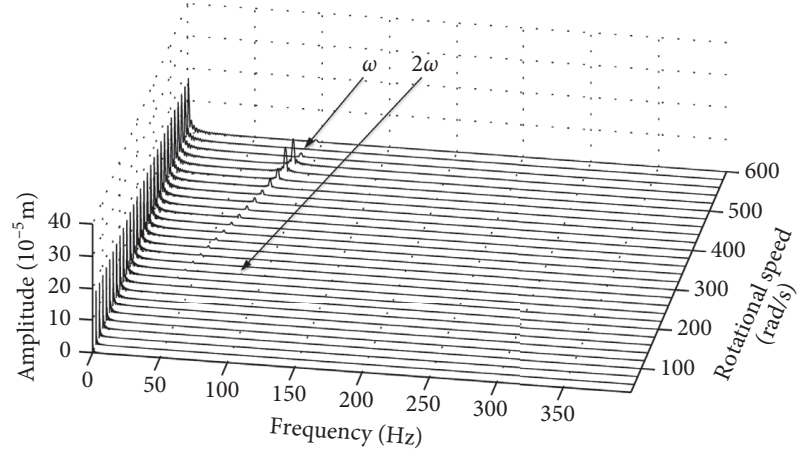

(a)

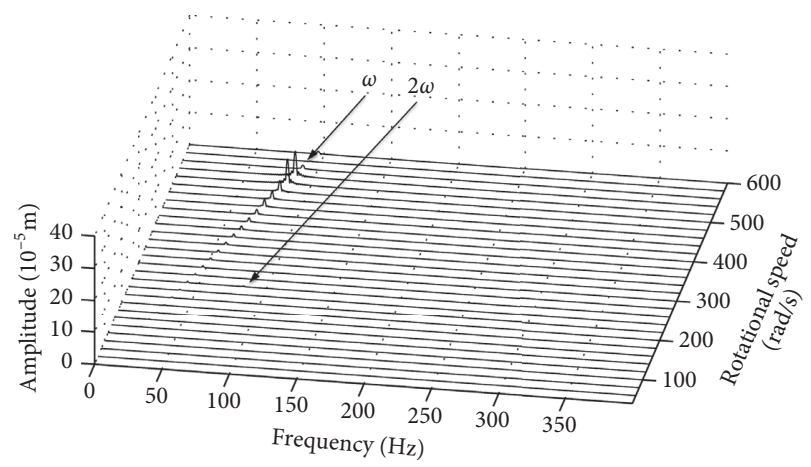

(c)

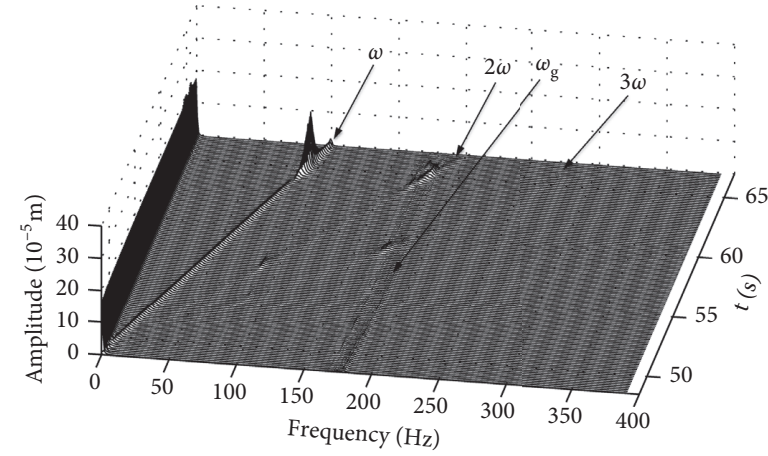

(b)

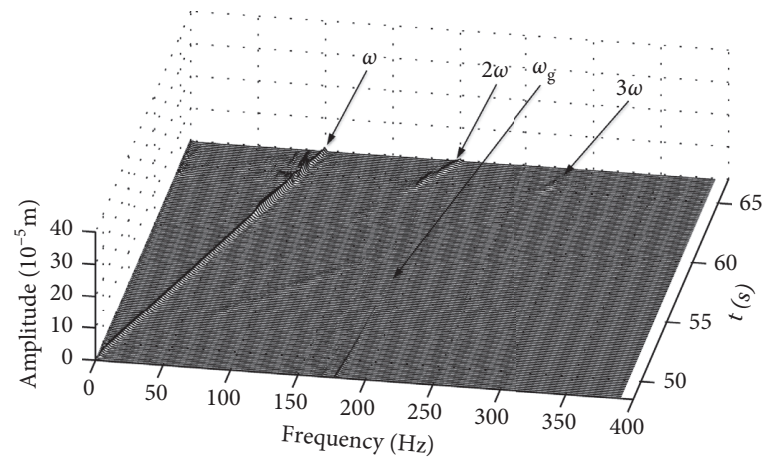

(d)

FIGURE 9: Waterfall spectra plots for the response of disk 3 during rolling. (a) Horizontal response, numerical simulation. (b) Horizontal response, experimental results. (c) Vertical response, numerical simulation. (d) Vertical response, experimental results.

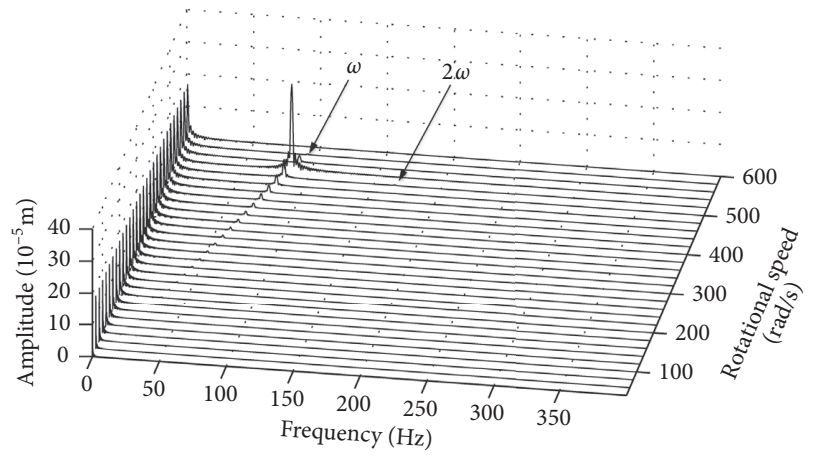

(a)

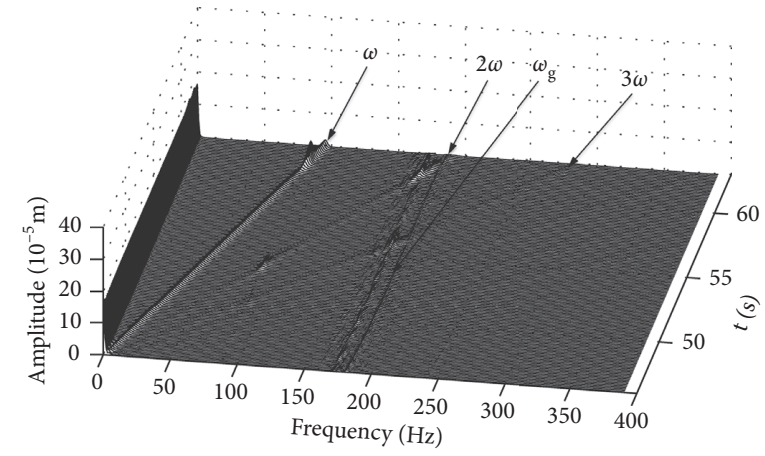

(b)

Figure 10: Continued. 


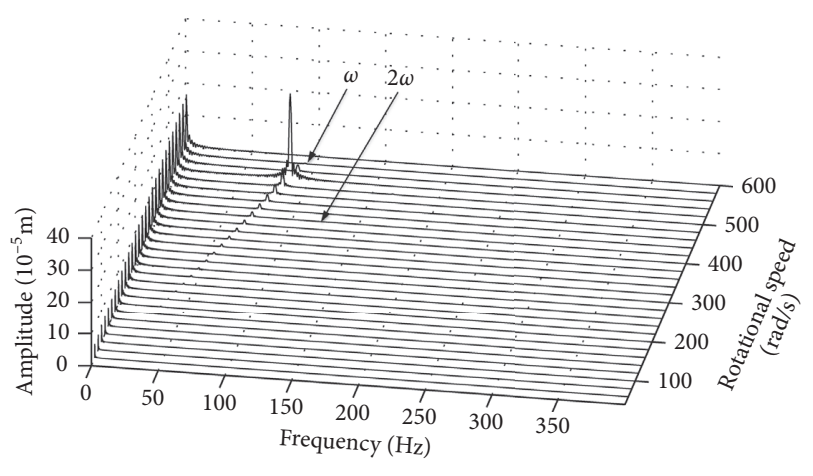

(c)

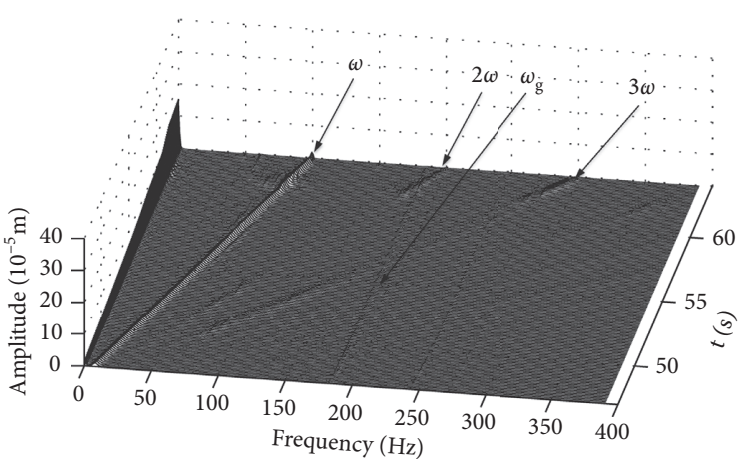

(d)

FIGURE 10: Waterfall spectra plots for the vibration of disk 3 during the synthetic maneuver of hovering and rolling. (a) Horizontal response, numerical simulation. (b) Horizontal response, experimental results. (c) Vertical response, numerical simulation. (d) Vertical response, experimental results.

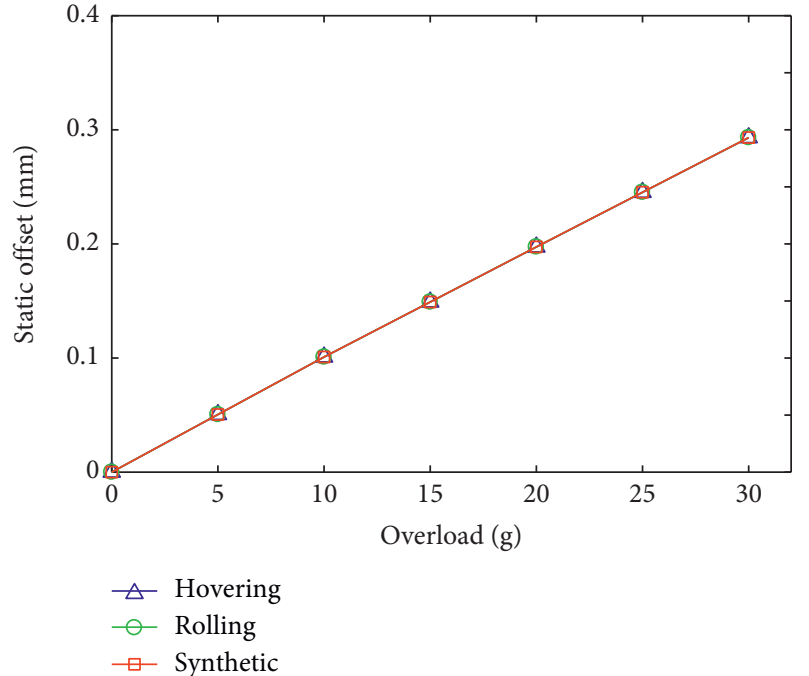

(a)

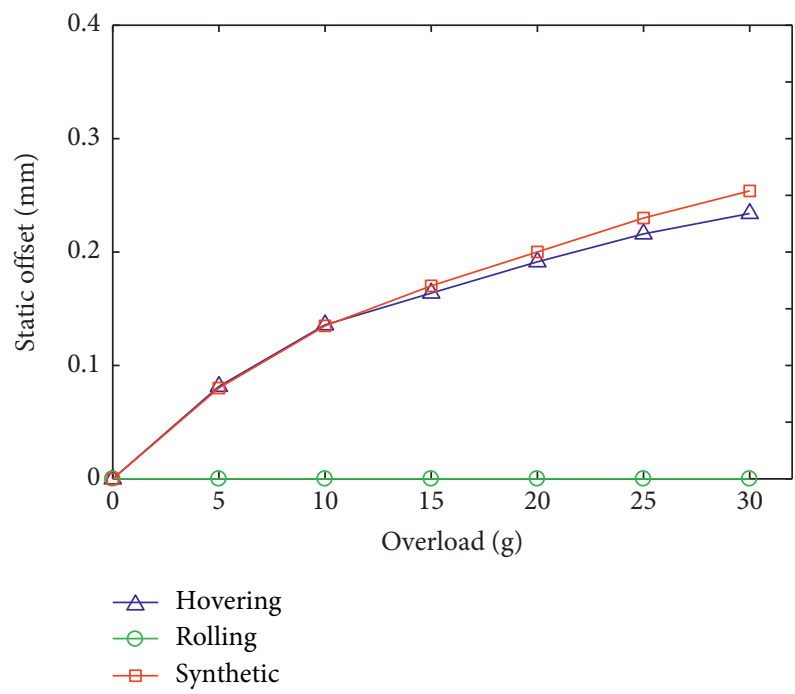

(c)

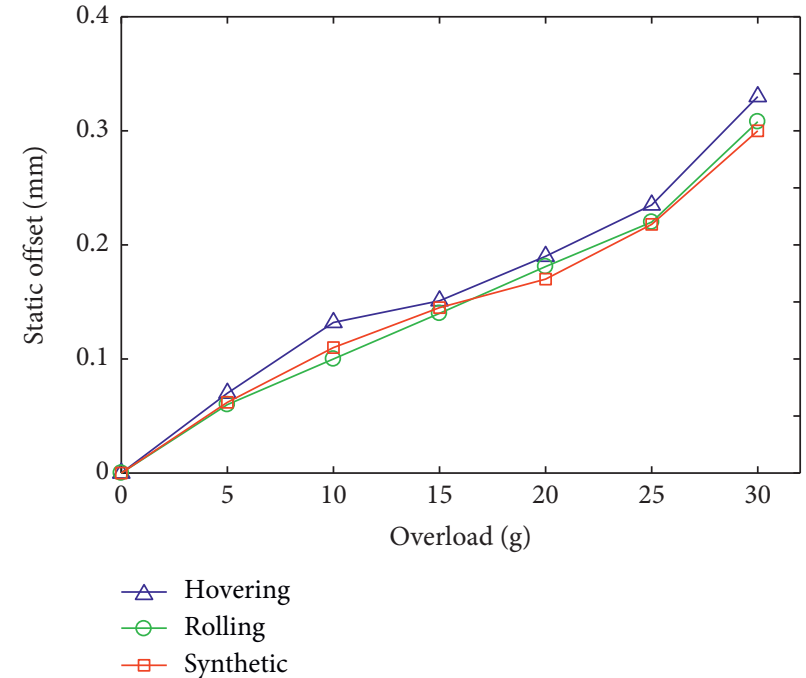

(b)

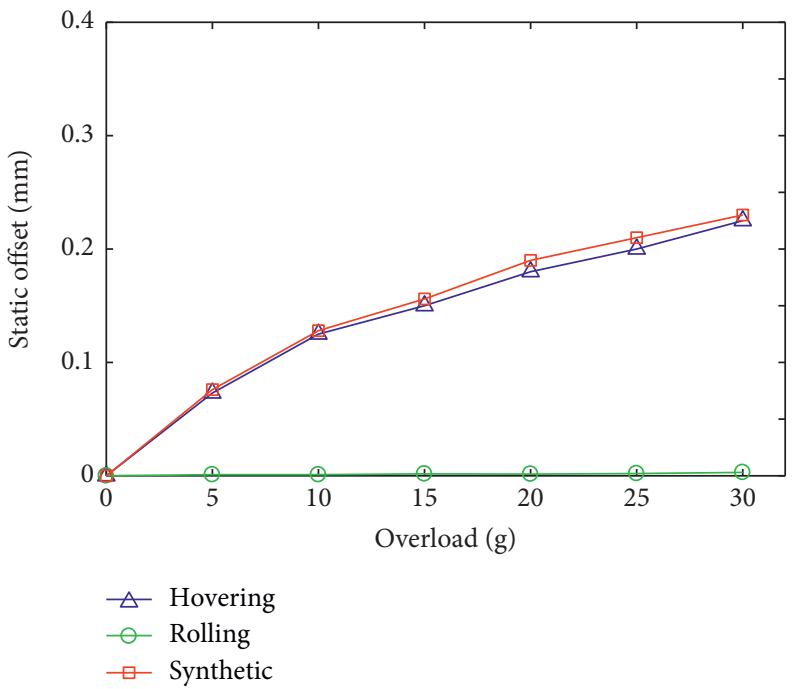

(d)

FIGURE 11: Variation result of the static displacements of disk 3 with overload under maneuver flight conditions. (a) Horizontal magnitude, numerical simulation. (b) Horizontal magnitude, experimental results. (c) Vertical magnitude, numerical simulation. (d) Vertical magnitude, experimental results. 


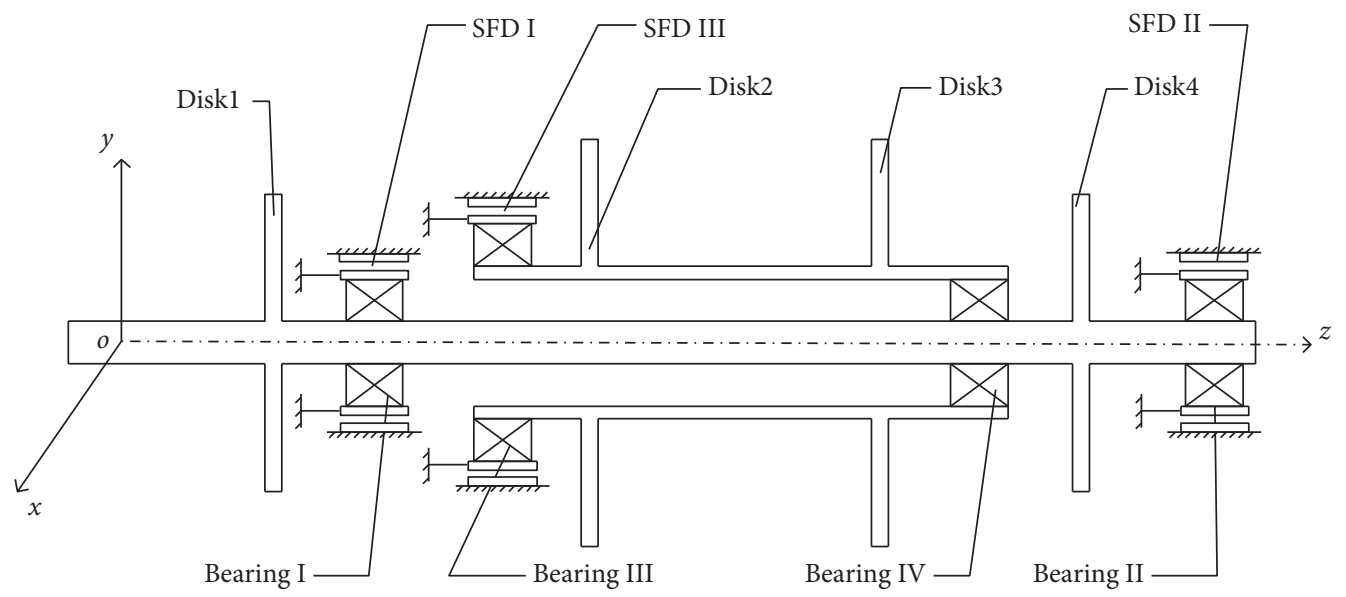

FIgURE 12: Structural diagram of the dual rotor system.

TABLE 5: Inertial properties and unbalance configuration of disks.

\begin{tabular}{|c|c|c|c|c|}
\hline \multirow{2}{*}{ Name } & \multicolumn{2}{|c|}{ Inner rotor } & \multicolumn{2}{|c|}{ Outer rotor } \\
\hline & Disk 1 & Disk 4 & Disk 2 & Disk 3 \\
\hline Mass (kg) & 2.41 & 2.38 & 3.17 & 1.57 \\
\hline Polar moment of inertia $\left(\mathrm{kg} \cdot \mathrm{m}^{2}\right)$ & 0.0082 & 0.0082 & 0.0146 & 0.0059 \\
\hline Unbalance $\left(10^{-5} \mathrm{~kg} \cdot \mathrm{m}\right)$ & 2 & 2 & 1.5 & 3 \\
\hline
\end{tabular}

TABLE 6: Radial stiffness of elastic supports.

\begin{tabular}{lccc}
\hline Name & I & II & III \\
\hline Stiffness $(\mathrm{N} / \mathrm{m})$ & $2.9 \times 10^{7}$ & $4.4 \times 10^{7}$ & $2 \times 10^{7}$ \\
\hline
\end{tabular}

TABLE 7: Parameters of SFDs.

\begin{tabular}{|c|c|c|c|}
\hline \multirow{2}{*}{ Name } & \multicolumn{2}{|c|}{ Inner rotor } & \multirow{2}{*}{$\begin{array}{c}\text { Outer rotor } \\
\text { SFD III }\end{array}$} \\
\hline & SFD I & SFD II & \\
\hline Radius (mm) & 25 & 18 & 35 \\
\hline Length (mm) & 13 & 13 & 14 \\
\hline Radial clearance $(\mathrm{mm})$ & 0.25 & 0.18 & 0.35 \\
\hline Dynamic viscosity $\left(10^{-2} \mathrm{~Pa} \cdot \mathrm{s}\right)$ & 1.0752 & - & - \\
\hline
\end{tabular}

overload of the centrifugal force can be adjusted through controlling the rotating speed of the rotating arm structure.

Figure 5 shows the bearing-rotor system, which is composed of a motor, a flexible coupling, three disks, and two supports. Rolling bearings are adopted for the supports, and the three disks contain two simply supported disks and one cantilever disk. Inertia properties and unbalance configuration of the disks are summarized in Table 1. Related parameters of bearings are provided in Table 2 .

To validate the correctness of the dynamic model, nine kinds of maneuver flight conditions are investigated by numerical simulation and experimental methods, respectively, as shown in Table 3, where $g$ denotes the gravitational acceleration. And the pitch angle is set to $30^{\circ}$ when the synthetic maneuver of hovering and rolling is studied.

3.2. Numerical and Experimental Results. The finite element model is established, which consists of 40 elements, including 35 beam elements, 3 disk elements, and 2 supporting elements, as shown in Figure 6. The geometric dimensions and information of each element are listed in Table 4.

The Newmark- $\beta$ method and Newton-Raphson method are used to solve the differential equations of motion. For the Newmark- $\beta$ method, two parameters are set as $\alpha=0.3$ and $\beta=0.6$. Without maneuver flight condition, the waterfall spectra plots for the vibration of disk 3 with the rotor operating in 20-600 rad/s using numerical simulation and experimental methods are shown in Figure 7. The comparison results manifest that the characteristics of the response obtained by numerical simulation and experimental approach agree well with each other. Compared with the numerical simulation results, the amplitudes of the double frequency and triple frequency components in the experimental test are more extensive due to the misalignment of shafting and coupling or asymmetry of the stiffness of bearings, which is not considered in the simulation calculation. 
TABLE 8: Parameters of the intermediate bearing.

\begin{tabular}{lcccc}
\hline Name & Radius of inner ring $(\mathrm{mm})$ & Radius of outer ring $(\mathrm{mm})$ & No. of rollers & Radial clearance $(\mu \mathrm{m})$ \\
\hline Value & 9.35 & 14.15 & 9 & 6 \\
\hline
\end{tabular}

Table 9: Dimension and elements information of the dual rotor system.

\begin{tabular}{|c|c|c|c|c|c|}
\hline Node number & Axial location (m) & Bearing/disk & Element number & Outer diameter $(\mathrm{m})$ & Inner diameter $(\mathrm{m})$ \\
\hline 1 & 0.100 & \multirow{12}{*}{ Disk 1} & 1 & 0.018 & 0 \\
\hline 2 & 0.130 & & 2 & 0.018 & 0 \\
\hline 3 & 0.160 & & 3 & 0.018 & 0 \\
\hline 4 & 0.190 & & 4 & 0.018 & 0 \\
\hline 5 & 0.220 & & 5 & 0.018 & 0 \\
\hline 6 & 0.255 & & 6 & 0.018 & 0 \\
\hline 7 & 0.289 & & 7 & 0.018 & 0 \\
\hline 8 & 0.323 & & 8 & 0.018 & 0 \\
\hline 9 & 0.359 & & 9 & 0.018 & 0 \\
\hline 10 & 0.384 & & 10 & 0.018 & 0 \\
\hline 11 & 0.409 & & 11 & 0.018 & 0 \\
\hline 12 & 0.433 & & 12 & 0.018 & 0 \\
\hline 13 & 0.445 & \multirow{8}{*}{ Bearing 1} & 13 & 0.018 & 0 \\
\hline 14 & 0.490 & & 14 & 0.022 & 0 \\
\hline 15 & 0.560 & & 15 & 0.022 & 0 \\
\hline 16 & 0.650 & & 16 & 0.022 & 0 \\
\hline 17 & 0.753 & & 17 & 0.022 & 0 \\
\hline 18 & 0.820 & & 18 & 0.022 & 0 \\
\hline 19 & 0.958 & & 19 & 0.022 & 0 \\
\hline 20 & 1.030 & & 20 & 0.022 & 0 \\
\hline 21 & 1.065 & \multirow{3}{*}{ Bearing 4} & 21 & 0.022 & 0 \\
\hline 22 & 1.087 & & 22 & 0.022 & 0 \\
\hline 23 & 1.100 & & 23 & 0.022 & 0 \\
\hline 24 & 1.140 & \multirow{4}{*}{ Disk 4} & 24 & 0.022 & 0 \\
\hline 25 & 1.170 & & 25 & 0.022 & 0 \\
\hline 26 & 1.190 & & 26 & 0.022 & 0 \\
\hline 27 & 1.211 & & 27 & 0.017 & 0 \\
\hline 28 & 1.215 & \multirow{6}{*}{ Bearing 2} & 28 & 0.014 & 0 \\
\hline 29 & 1.220 & & 29 & 0.014 & 0 \\
\hline 30 & 1.230 & & End of the inner rotor & & \\
\hline 31 & 0.642 & & 30 & 0.035 & 0.03 \\
\hline 32 & 0.655 & & 31 & 0.035 & 0.03 \\
\hline 33 & 0.679 & & 32 & 0.035 & 0.03 \\
\hline 34 & 0.687 & \multirow{3}{*}{ Bearing 3} & 33 & 0.038 & 0.03 \\
\hline 35 & 0.711 & & 34 & 0.038 & 0.03 \\
\hline 36 & 0.737 & & 35 & 0.038 & 0.03 \\
\hline 37 & 0.762 & \multirow{5}{*}{ Disk 2} & 36 & 0.038 & 0.03 \\
\hline 38 & 0.808 & & 37 & 0.038 & 0.03 \\
\hline 39 & 0.854 & & 38 & 0.038 & 0.03 \\
\hline 40 & 0.899 & & 39 & 0.038 & 0.03 \\
\hline 41 & 0.945 & & 40 & 0.038 & 0.03 \\
\hline 42 & 0.960 & \multirow{5}{*}{ Disk 3} & 41 & 0.038 & 0.03 \\
\hline 43 & 0.980 & & 42 & 0.038 & 0.03 \\
\hline 44 & 1.000 & & 43 & 0.07 & 0.03 \\
\hline 45 & 1.020 & & 44 & 0.06 & 0.03 \\
\hline 46 & 1.040 & & 45 & 0.06 & 0.03 \\
\hline 47 & 1.064 & \multirow{2}{*}{ Bearing 4} & 46 & 0.06 & 0.03 \\
\hline 48 & 1.074 & & & & \\
\hline
\end{tabular}

TABle 10: Parameters of maneuver flight.

\begin{tabular}{lccc}
\hline Maneuvers & $v_{B, z}(\mathrm{~m} / \mathrm{s})$ & $\omega_{B, y}(\mathrm{rad} / \mathrm{s})$ & Overload $(\mathrm{g})$ \\
\hline Horizontal turn & 98 & 1 & 10 \\
\hline
\end{tabular}

The waterfall spectra plots for the vibration of disk 3 with the rotor operating in 20-600 rad/s under the maneuver flight conditions of hovering, rolling, and the synthetic maneuver of hovering and rolling with the overload of $20 \mathrm{~g}$ 


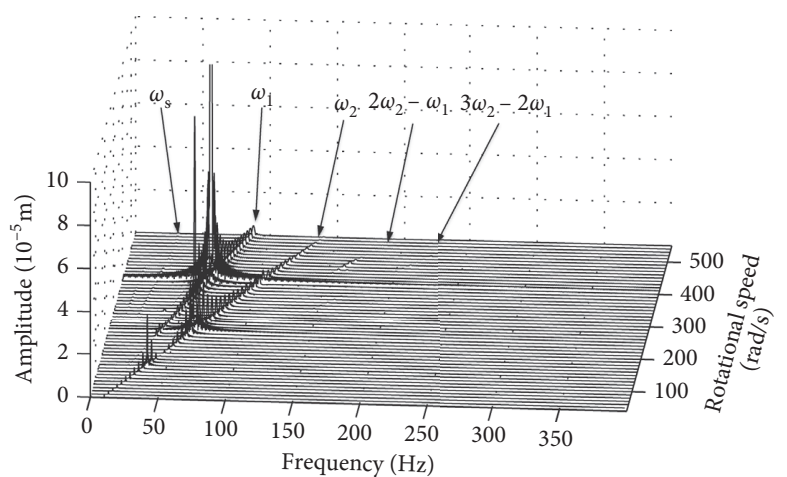

(a)

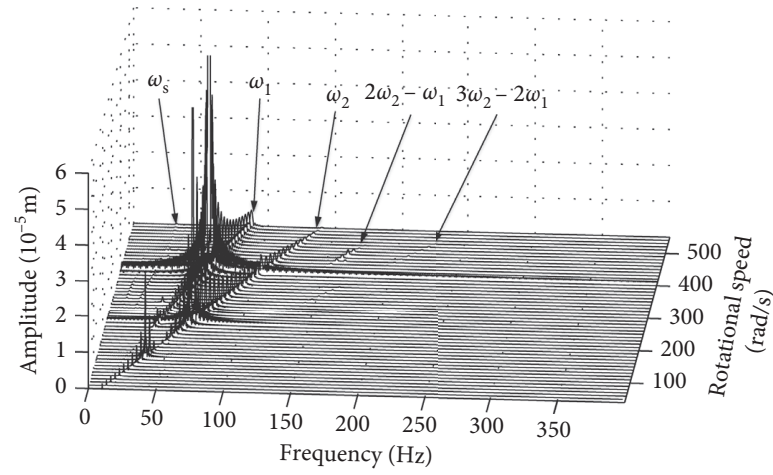

(c)

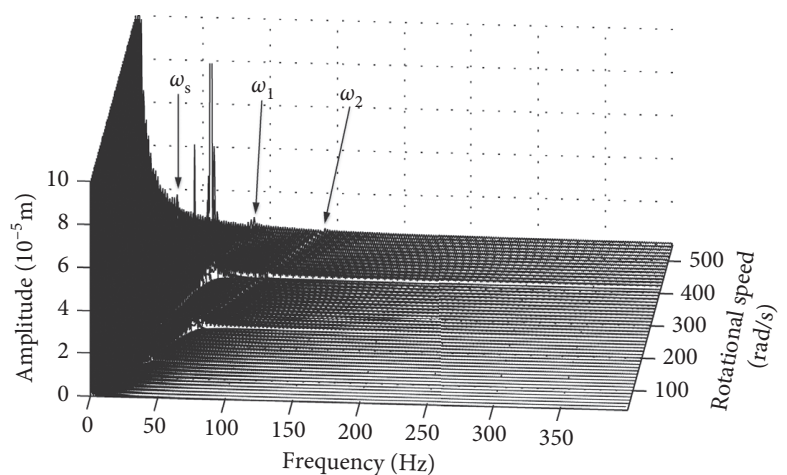

(b)

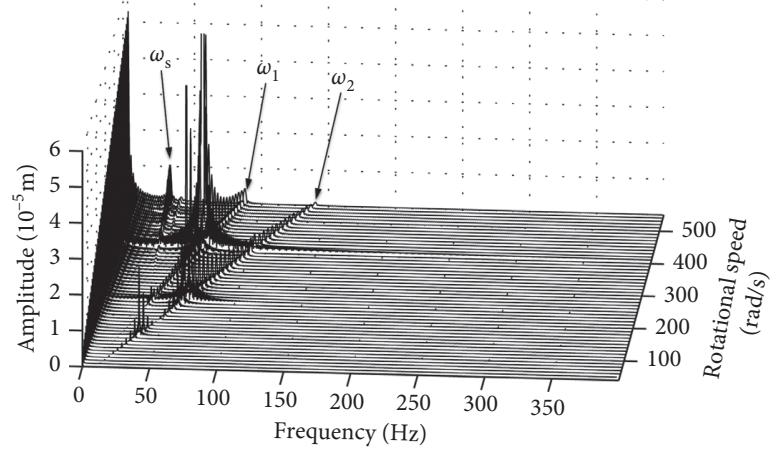

(d)

FIGURE 13: Waterfall spectra plots for the vibration of disk 3. (a) Horizontal response, straight flight. (b) Horizontal response, hovering. (c) Vertical response, straight flight. (d) Vertical response, hovering.

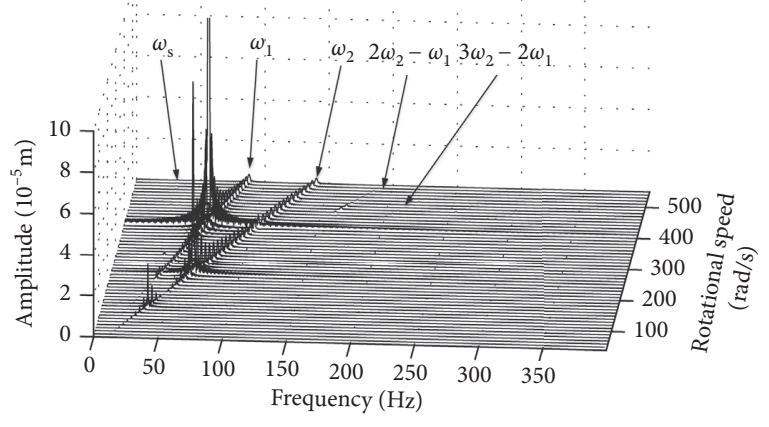

(a)

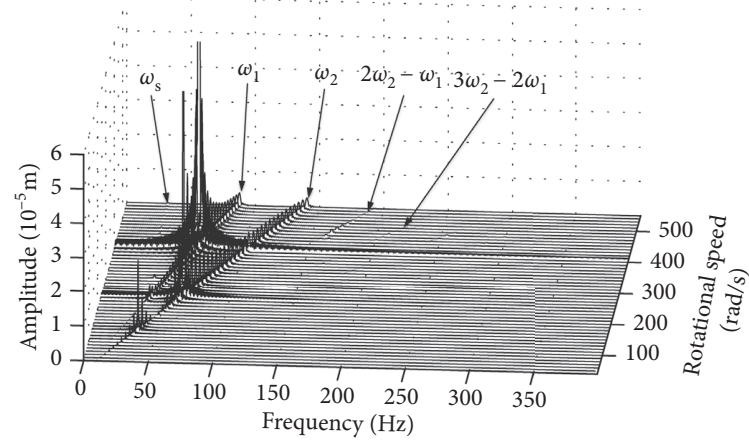

(c)

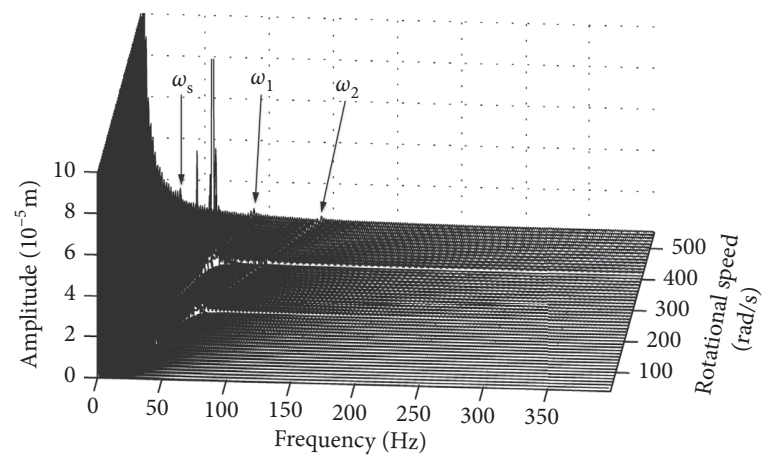

(b)

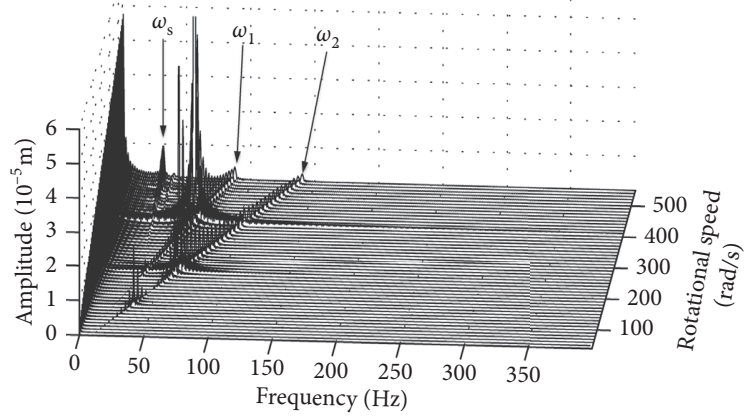

(d)

FIgURE 14: Waterfall spectra plots for the vibration of disk 4. (a) Horizontal response, straight flight. (b) Horizontal response, hovering. (c) Vertical response, straight flight. (d) Vertical response, hovering. 


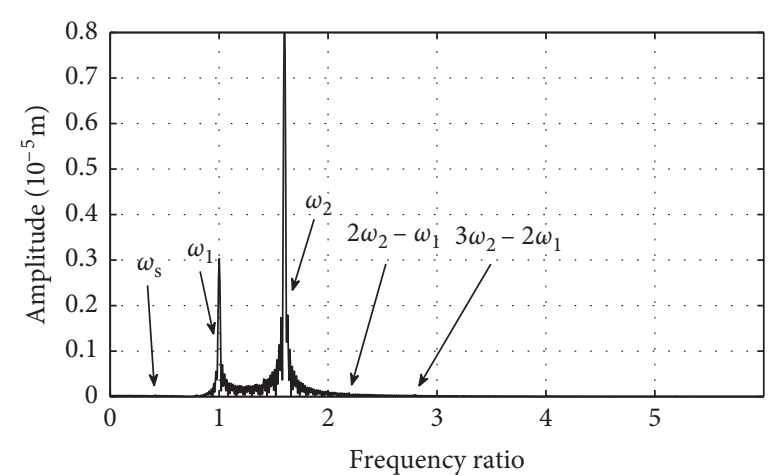

(a)

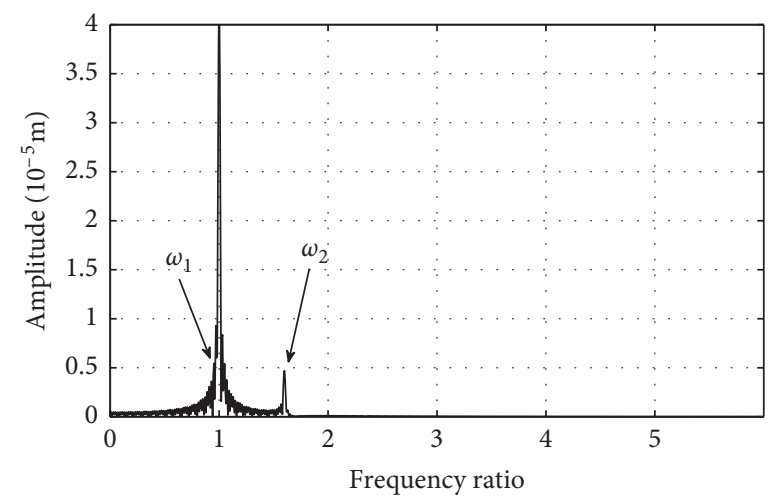

(c)

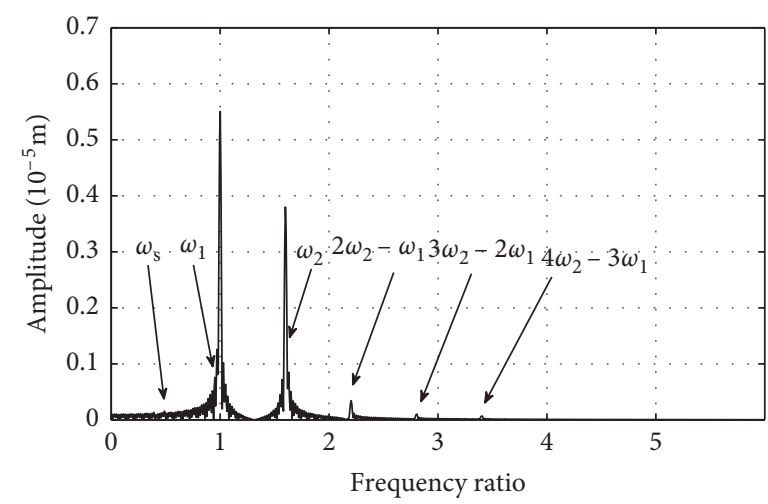

(e)

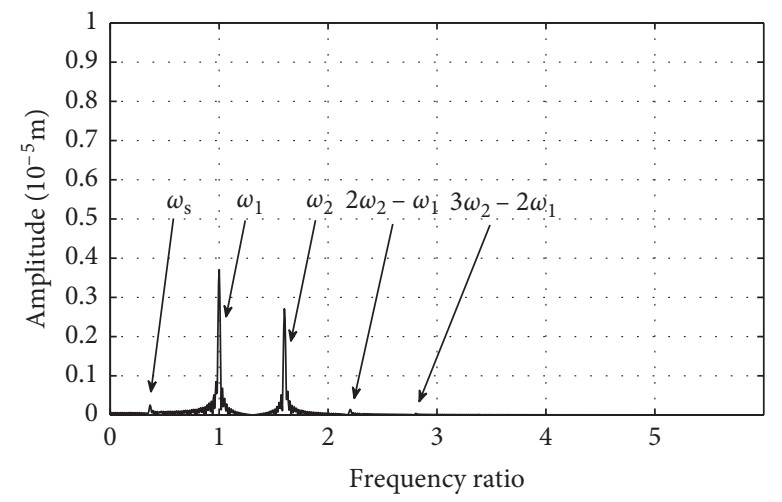

(g)

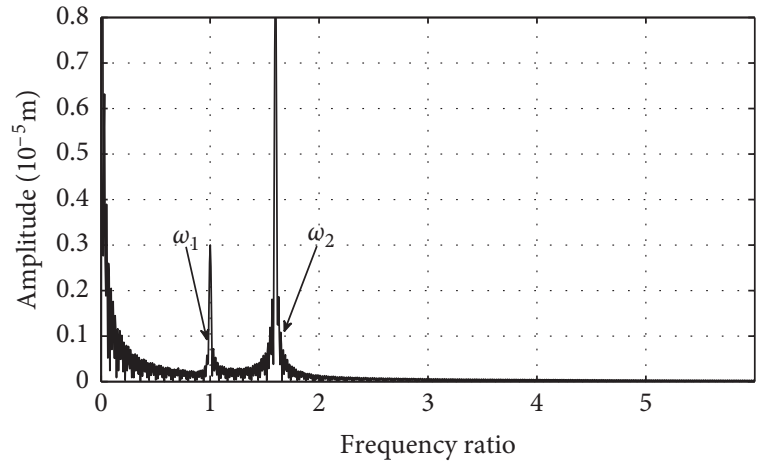

(b)

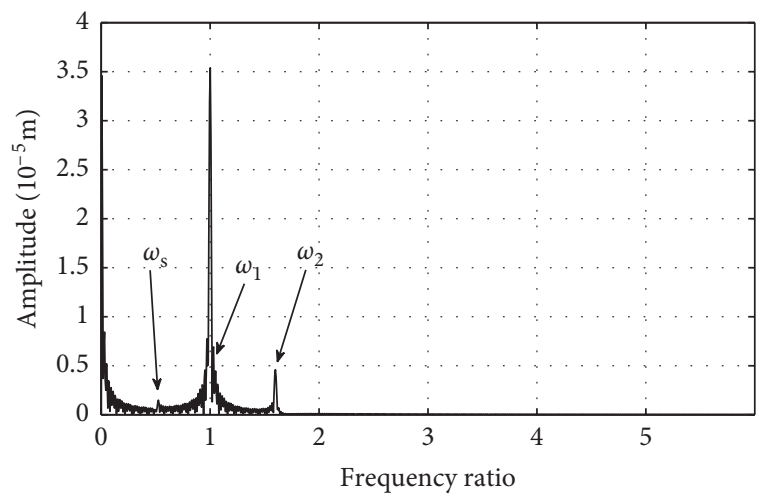

(d)

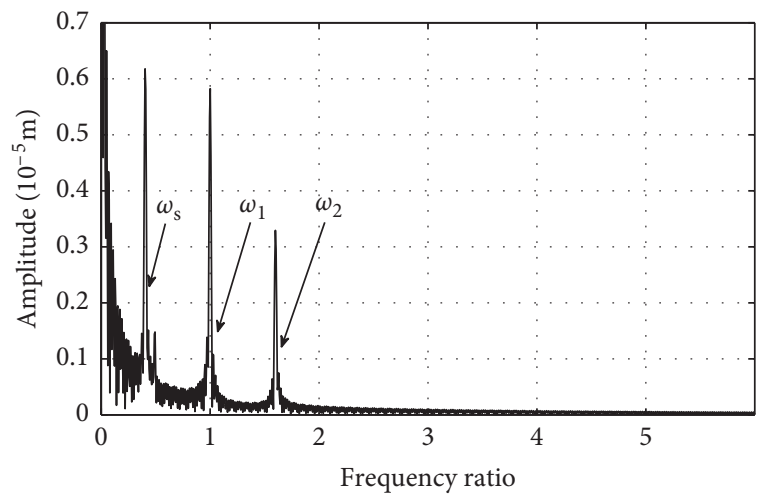

(f)

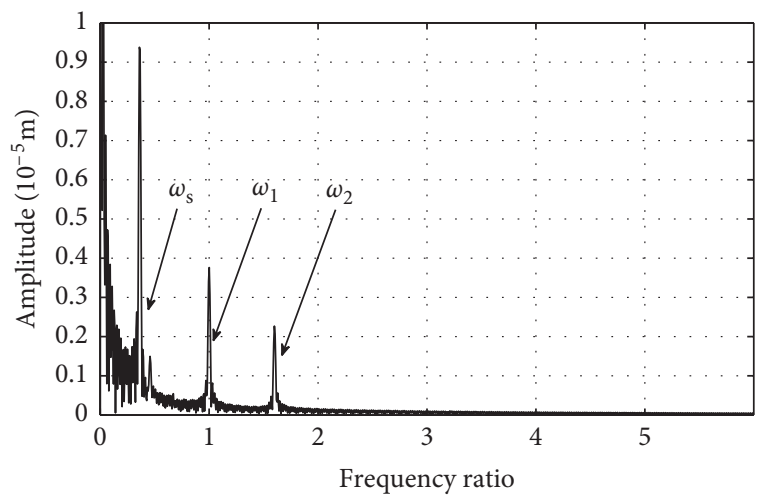

(h)

FIGURE 15: Spectrograms for the vertical vibration of disk 4. (a) $300 \mathrm{rad} / \mathrm{s}$, straight flight. (b) $300 \mathrm{rad} / \mathrm{s}$, hovering. (c) $400 \mathrm{rad} / \mathrm{s}$, straight flight. (d) $400 \mathrm{rad} / \mathrm{s}$, hovering. (e) $500 \mathrm{rad} / \mathrm{s}$, straight flight. (f) $500 \mathrm{rad} / \mathrm{s}$, hovering. (g) $550 \mathrm{rad} / \mathrm{s}$, straight flight. (h) $550 \mathrm{rad} / \mathrm{s}$, hovering. 


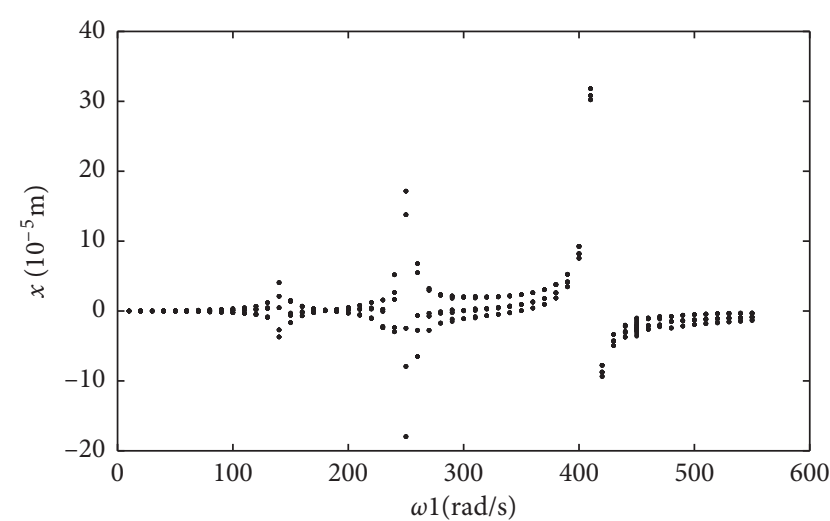

(a)

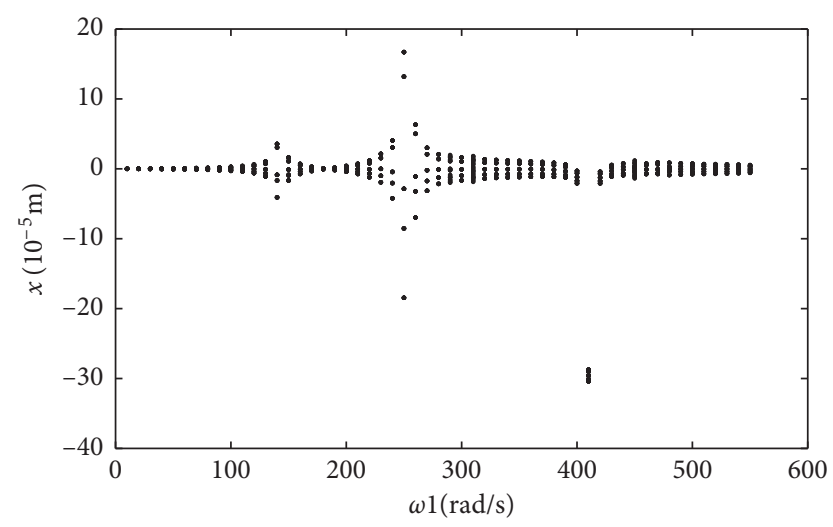

(c)

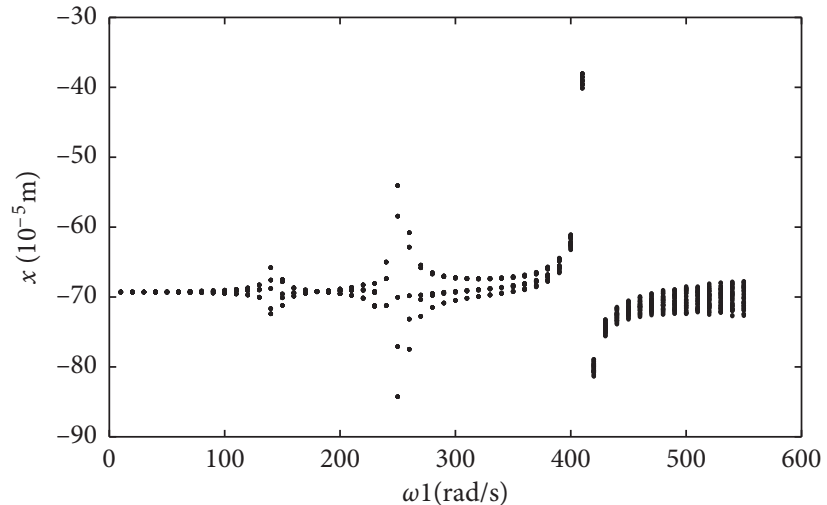

(b)

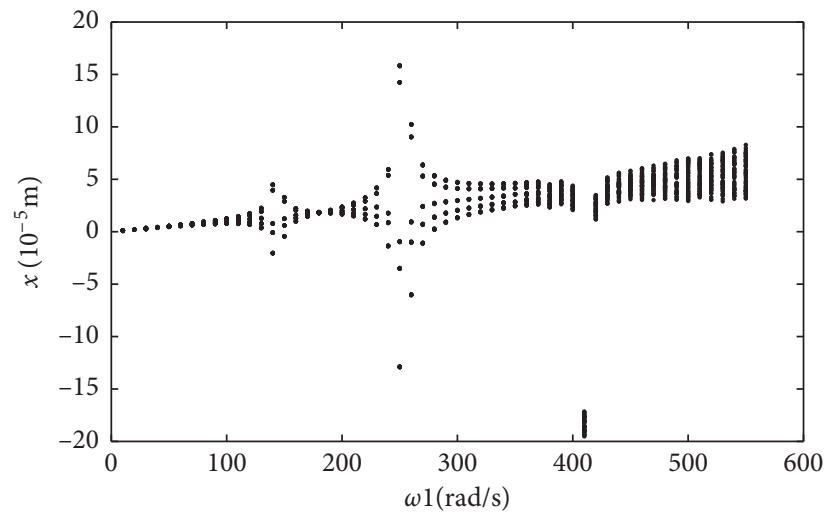

(d)

FIGURE 16: Bifurcation diagrams for the vibration of disk 4. (a) Horizontal response, straight flight. (b) Horizontal response, hovering. (c) Vertical response, straight flight. (d) Vertical response, hovering.

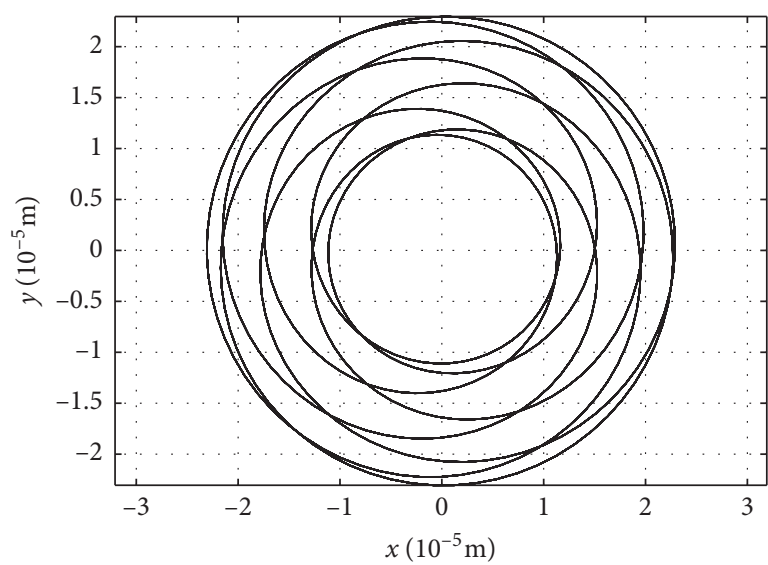

(a)

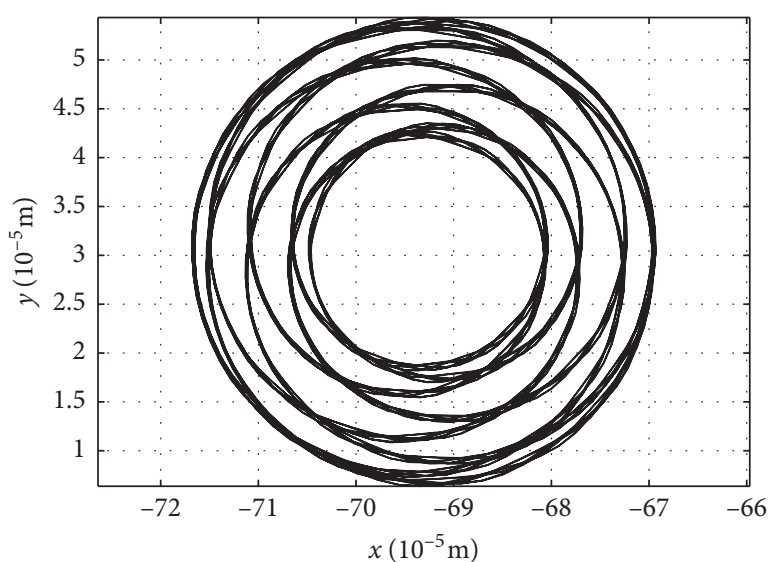

(b)

Figure 17: Continued. 


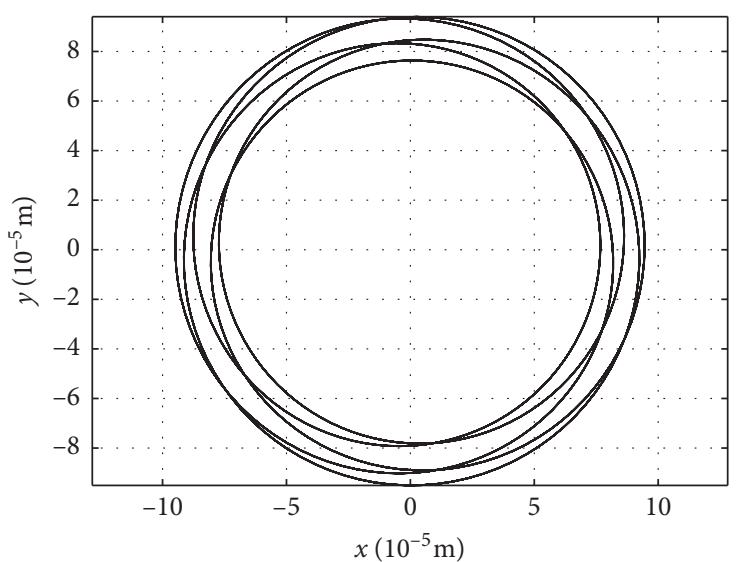

(c)

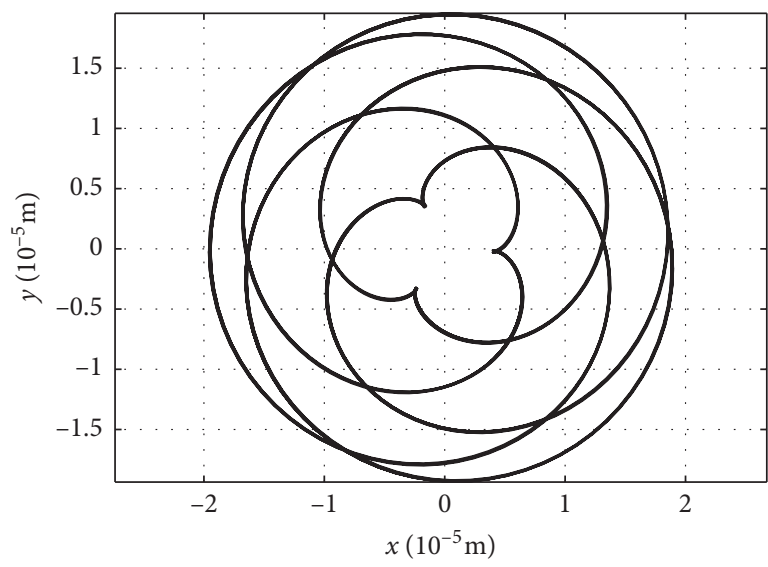

(e)

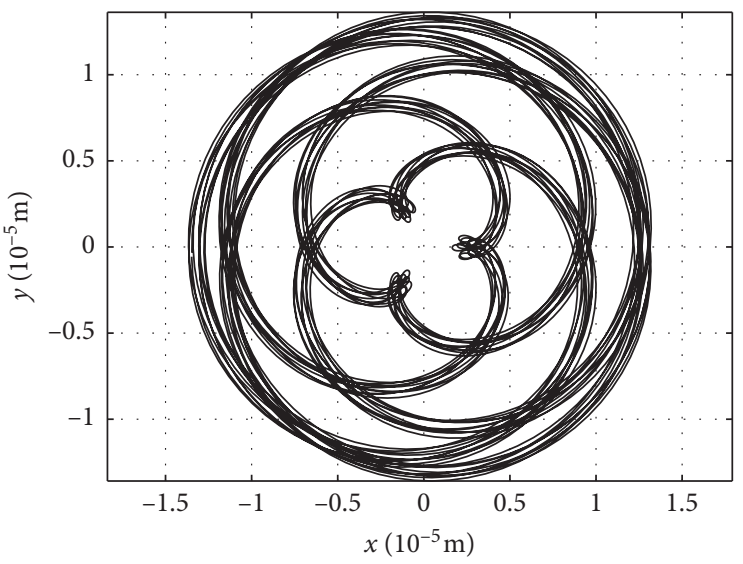

(g)

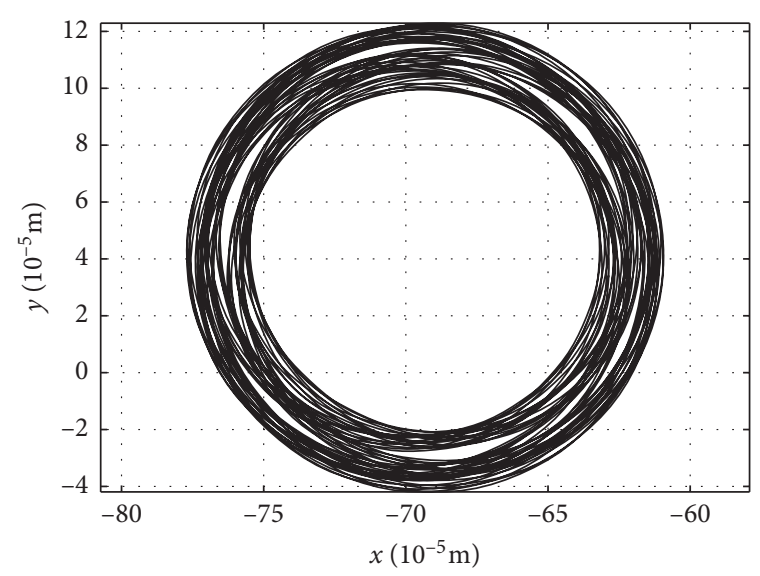

(d)

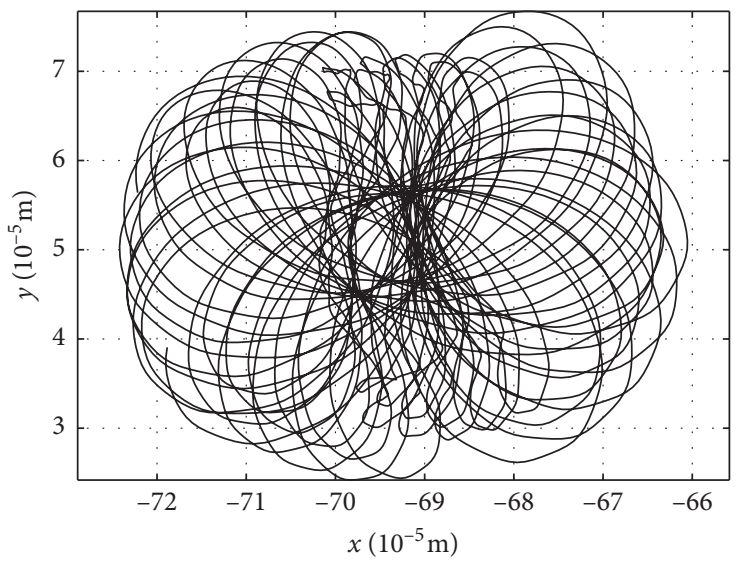

(f)

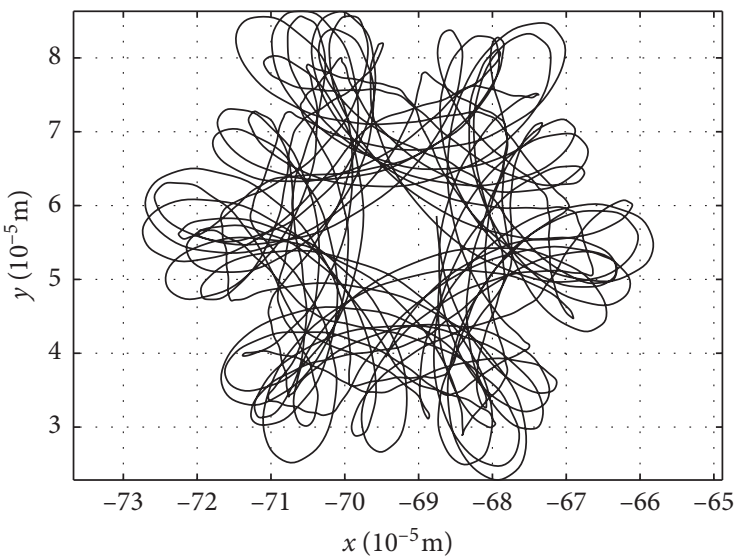

(h)

Figure 17: Orbit of disk 4. (a) $300 \mathrm{rad} / \mathrm{s}$, straight flight. (b) $300 \mathrm{rad} / \mathrm{s}$, hovering. (c) $400 \mathrm{rad} / \mathrm{s}$, straight flight. (d) $400 \mathrm{rad} / \mathrm{s}$, hovering. (e) $500 \mathrm{rad} / \mathrm{s}$, straight flight. (f) $500 \mathrm{rad} / \mathrm{s}$, hovering. (g) $550 \mathrm{rad} / \mathrm{s}$, straight flight. (h) $550 \mathrm{rad} / \mathrm{s}$, hovering.

are shown in Figures 8-10, respectively. The frequency component $\omega_{g}$ in experimental results is induced by the internal gear transmission in the reduction gearbox, which is not discussed in this work. By comparing the vibration response under conditions of straight flight and maneuver flight, a zero-frequency vibration component is observed during maneuver flight; that is, the static displacements of the rotor are generated under constant maneuvering overload. The static displacements in the horizontal direction caused by the additional centrifugal force do not vary with the rotating speed of the rotor. And the static displacements in the vertical direction induced by the additional gyro moment rise as the rotating speed increases. Especially, when the aircraft makes the flight maneuver of rolling, the rotation direction of the rotor is the same as its revolution. No maneuvering gyro torque is generated; 


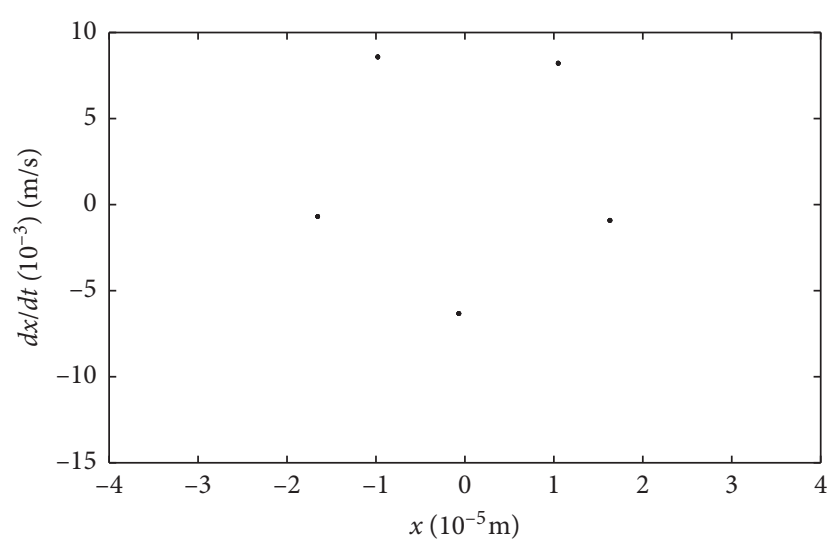

(a)

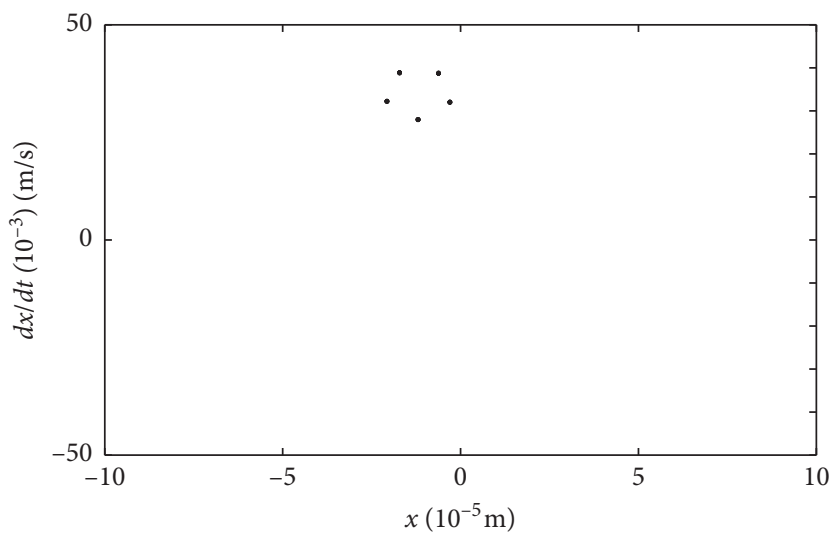

(c)

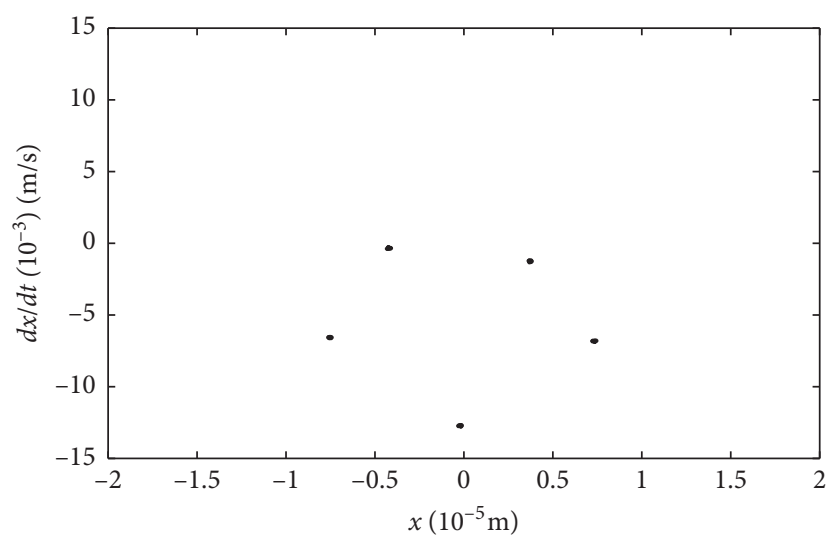

(e)

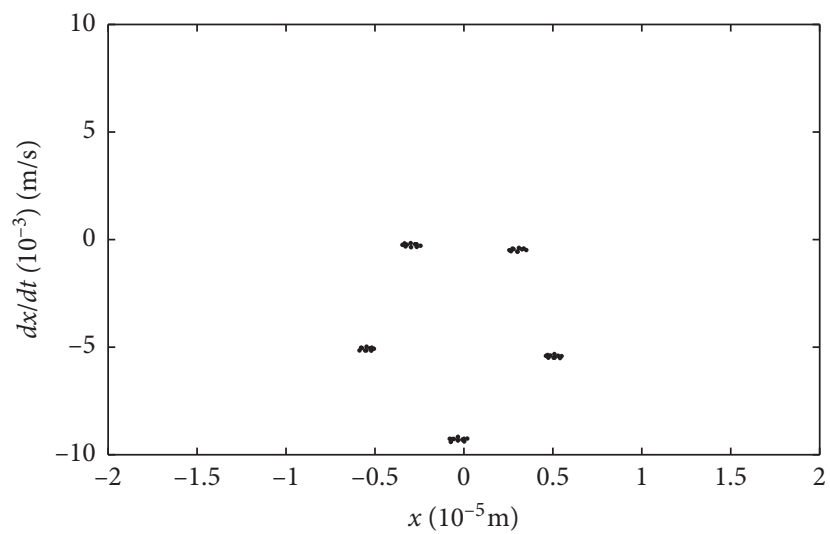

(g)

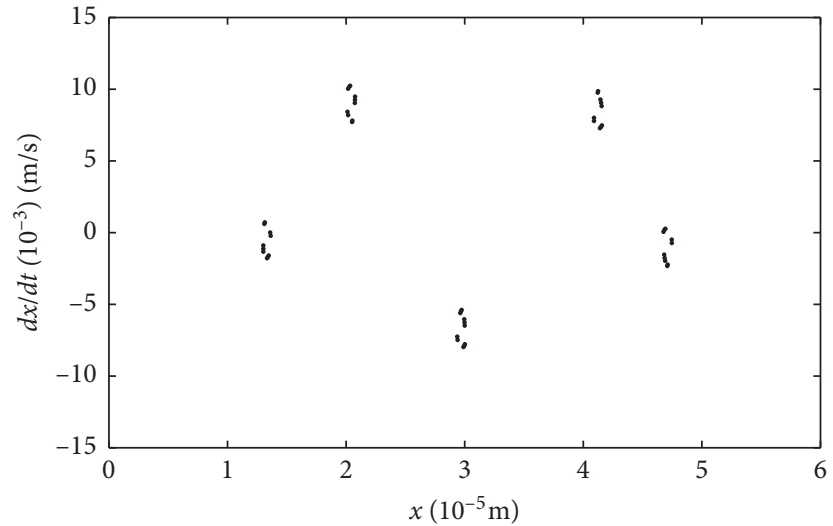

(b)

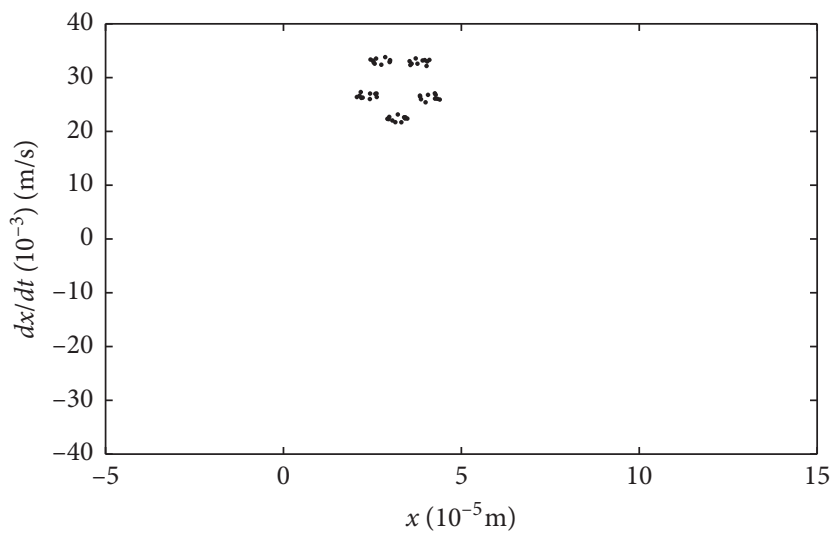

(d)

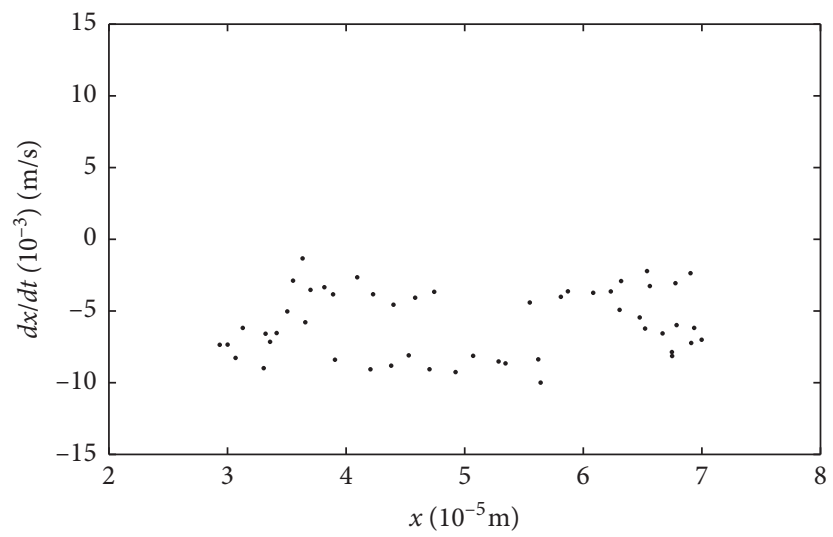

(f)

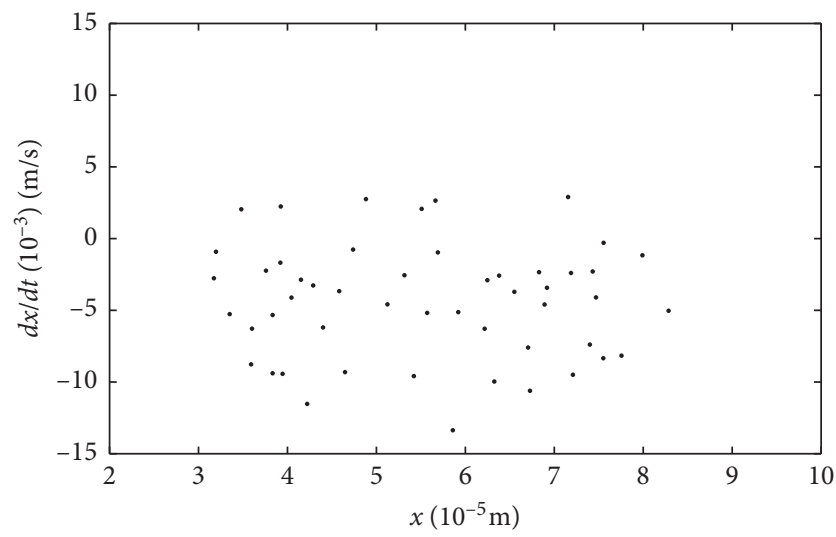

(h)

FIGURE 18: Poincare maps of disk 4. (a) $300 \mathrm{rad} / \mathrm{s}$, straight flight. (b) $300 \mathrm{rad} / \mathrm{s}$, hovering. (c) $400 \mathrm{rad} / \mathrm{s}$, straight flight. (d) $400 \mathrm{rad} / \mathrm{s}$, hovering. (e) $500 \mathrm{rad} / \mathrm{s}$, straight flight. (f) $500 \mathrm{rad} / \mathrm{s}$, hovering. (g) $550 \mathrm{rad} / \mathrm{s}$, straight flight. (h) $550 \mathrm{rad} / \mathrm{s}$, hovering. 


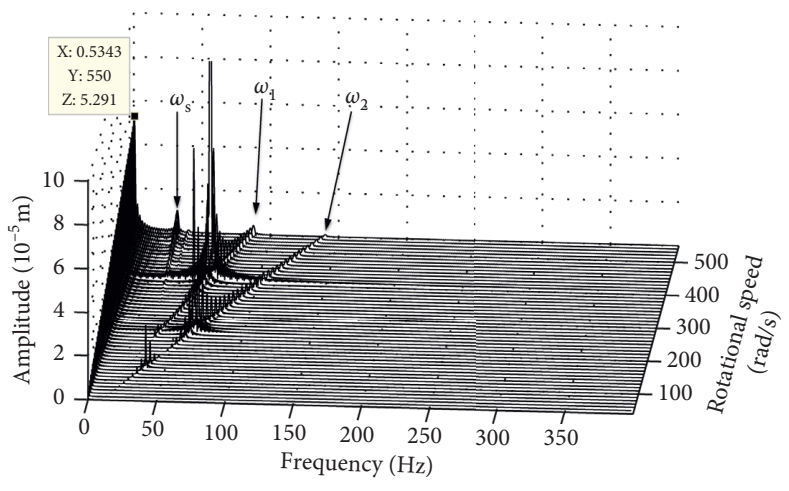

(a)

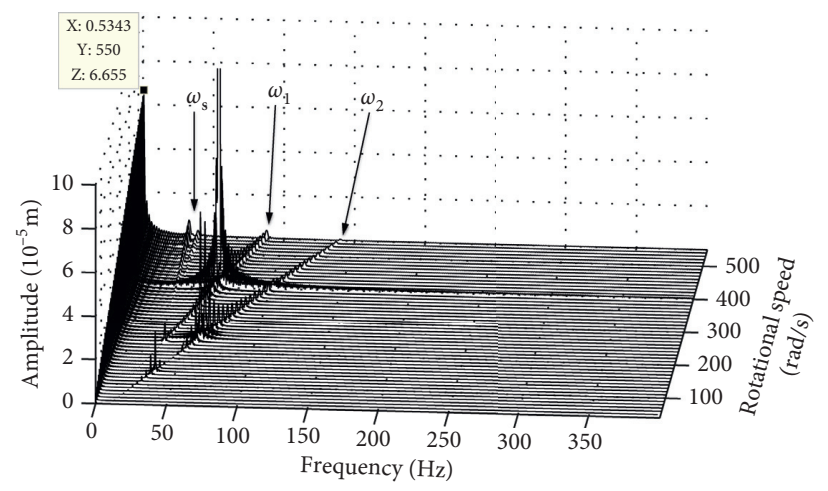

(b)

Figure 19: Vertical response of disk 3. (a) $\lambda=1.6$. (b) $\lambda=-1.6$.

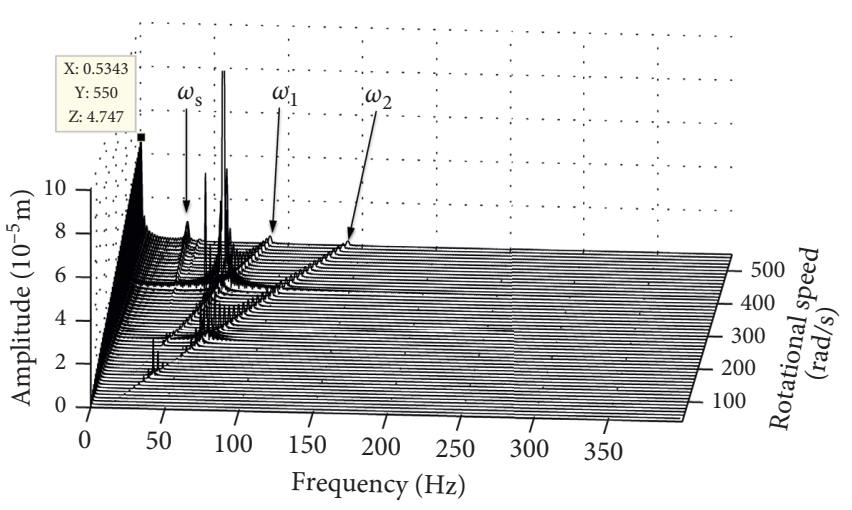

(a)

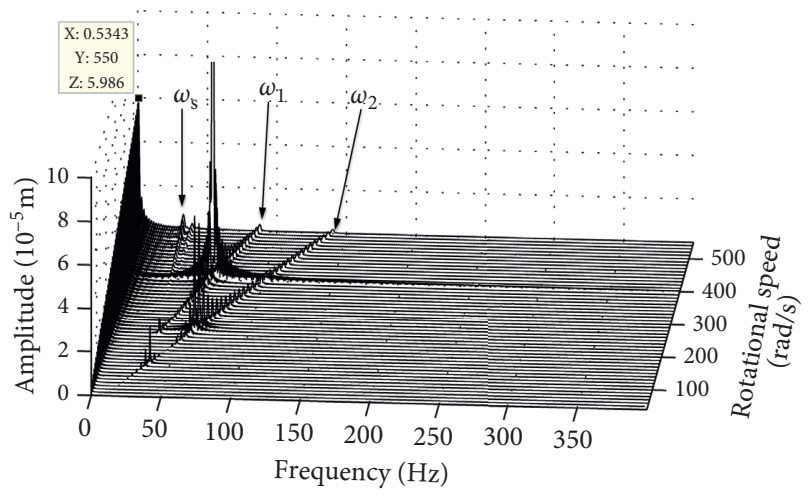

(b)

Figure 20: Vertical response of disk 4. (a) $\lambda=1.6$. (b) $\lambda=-1.6$.

therefore, there is no static displacement in the vertical direction, as shown in Figures 9(c) and 9(d).

With comparison of the influence of maneuver flight actions on the magnitude of the static displacements in numerical simulation and experimental works, Figure 11 shows the variation of the static displacements of disk 3 with the amount of overload under the maneuver flight conditions of hovering, rolling, and the synthetic maneuver when the rotor operates at $600 \mathrm{rad} / \mathrm{s}$. It can be observed that the magnitude of the static displacements through numerical simulation and experimental methods is basically consistent.

According to the above result analysis, the dynamic model of the bearing-rotor system under arbitrary maneuver flight conditions presented in this paper has the superior ability to predict the dynamic behavior of the rotor system under constant maneuvering overload.

\section{Complex Dual Rotor System}

The dynamic model of the bearing-rotor system under arbitrary maneuver flight conditions has been established and verified by experimental works. Based on this, the dynamic behavior of a complex intermediate bearing-SFD-rotor system under constant maneuvering overload is investigated using the finite element model proposed in this paper.

4.1. Nonlinear Forces of the Supports. The nonlinear oil film forces of SFD in the horizontal and vertical directions can be obtained by Reynolds equation [21, 22], which are presented as follows:

$$
\left\{\begin{aligned}
f_{x}^{s f d} & =-\frac{\mu R L^{3}}{c^{2}\left(x^{2}+y^{2}\right)^{(1 / 2)}}\left[x\left(\dot{\varepsilon} I_{2}+\varepsilon \dot{\psi} I_{1}\right)+y\left(\dot{\varepsilon} I_{1}+\varepsilon \dot{\psi} I_{3}\right)\right] \\
f_{y}^{s f d} & =-\frac{\mu R L^{3}}{c^{2}\left(x^{2}+y^{2}\right)^{(1 / 2)}}\left[y\left(\dot{\varepsilon} I_{2}+\varepsilon \dot{\psi} I_{1}\right)-x\left(\dot{\varepsilon} I_{1}+\varepsilon \dot{\psi} I_{3}\right)\right] \\
\varepsilon & =\frac{\sqrt{x^{2}+y^{2}}}{c} \\
\dot{\varepsilon} & =\frac{x \dot{x}+y \dot{y}}{c \sqrt{x^{2}+y^{2}}}
\end{aligned}\right.
$$




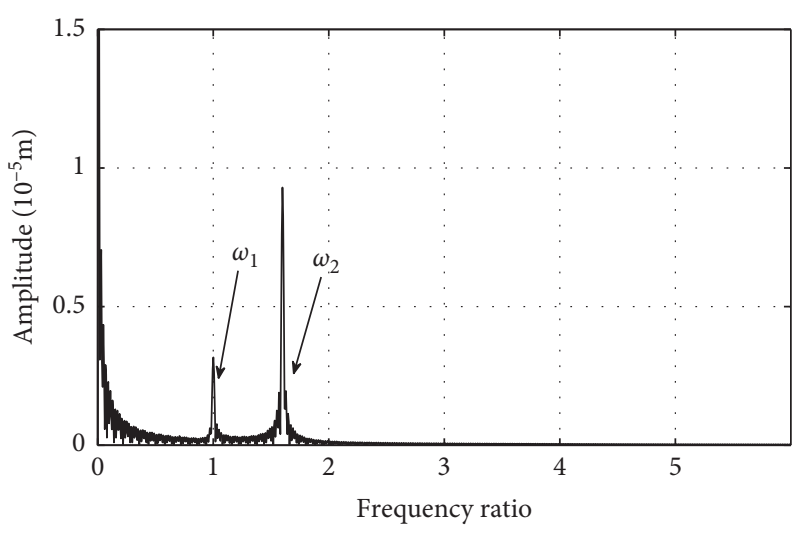

(a)

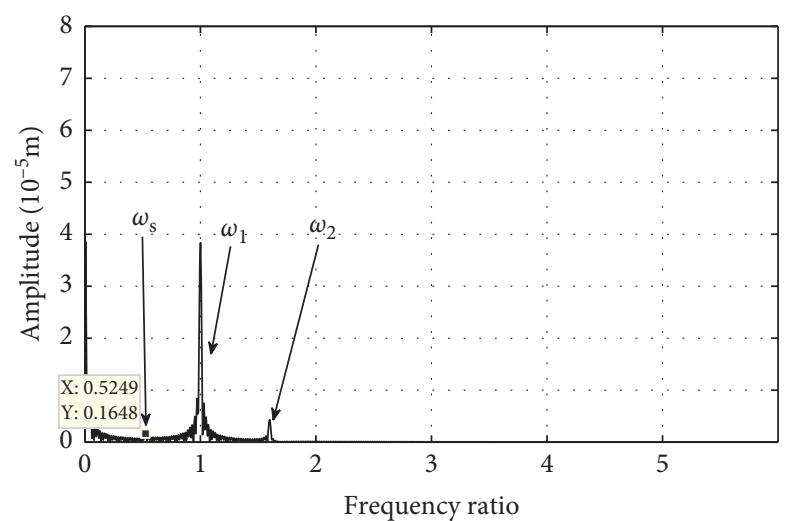

(c)

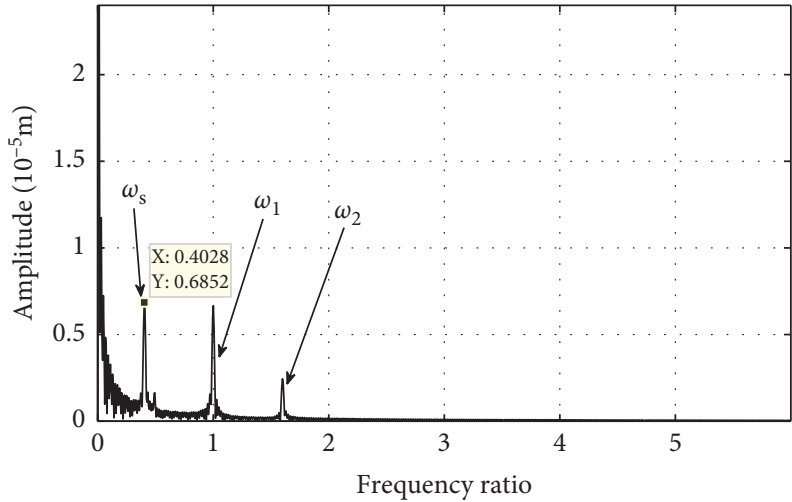

(e)

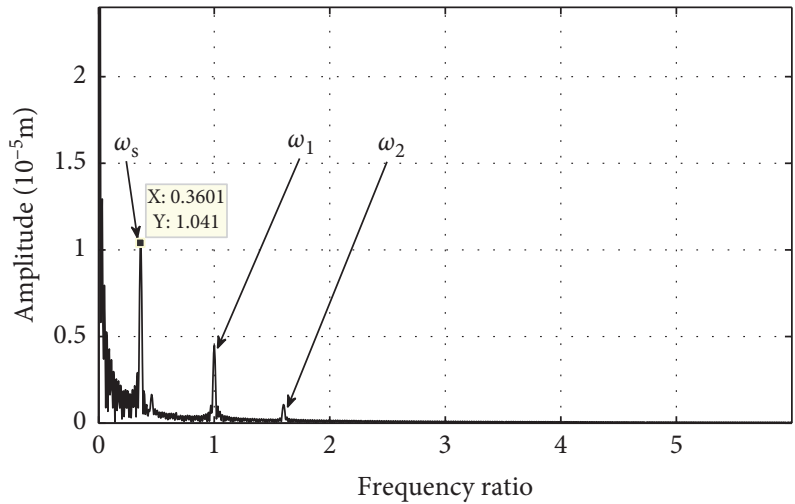

(g)

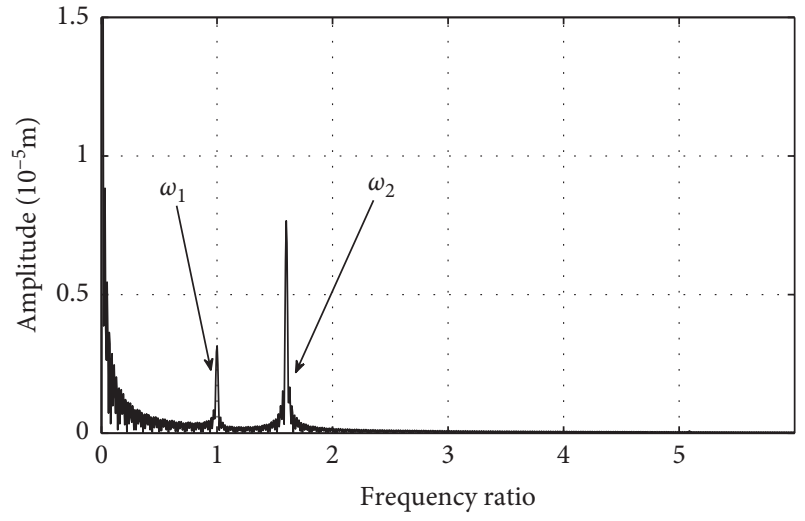

(b)

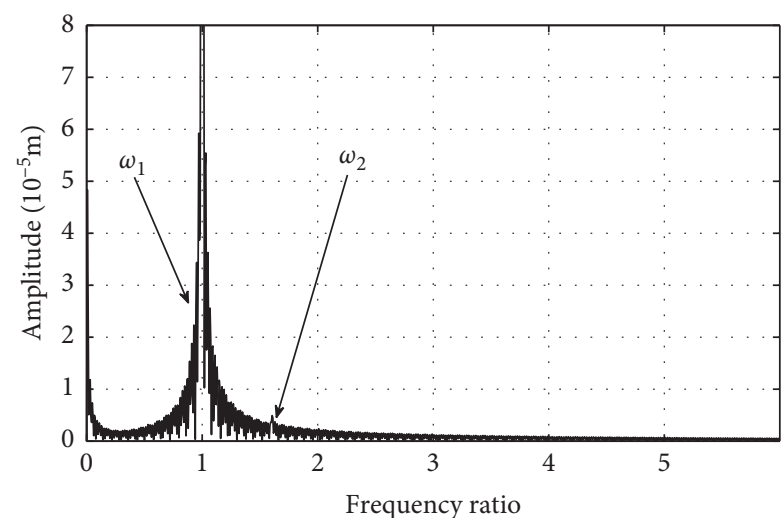

(d)

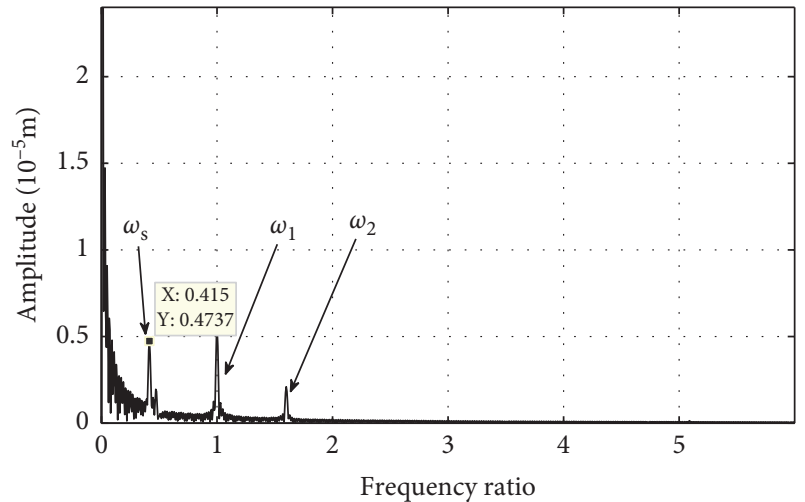

(f)

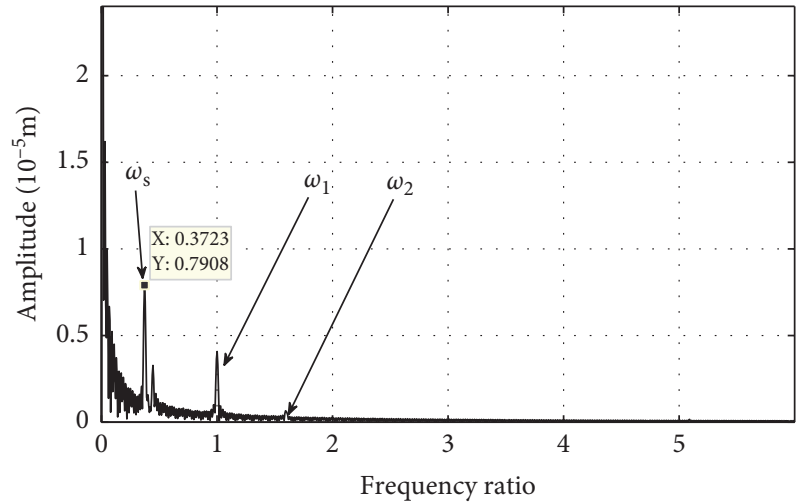

(h)

Figure 21: Spectrograms for the vertical vibration of disk 3. (a) $300 \mathrm{rad} / \mathrm{s}, \lambda=1.6$. (b) $300 \mathrm{rad} / \mathrm{s}, \lambda=-1.6$. (c) $400 \mathrm{rad} / \mathrm{s}, \lambda=1.6$. (d) $400 \mathrm{rad} / \mathrm{s}$, $\lambda=-1.6$. (e) $500 \mathrm{rad} / \mathrm{s}, \lambda=1.6$. (f) $500 \mathrm{rad} / \mathrm{s}, \lambda=-1.6$. (g) $550 \mathrm{rad} / \mathrm{s}, \lambda=1.6$. (h) $550 \mathrm{rad} / \mathrm{s}, \lambda=-1.6$. 


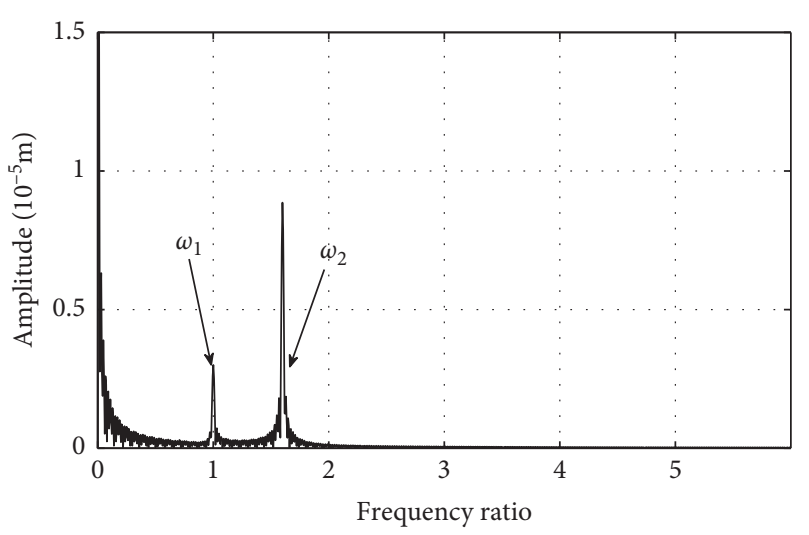

(a)

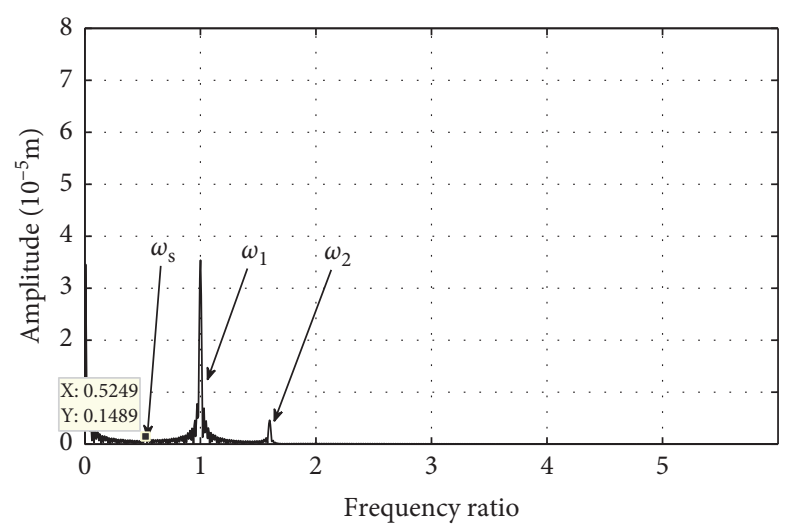

(c)

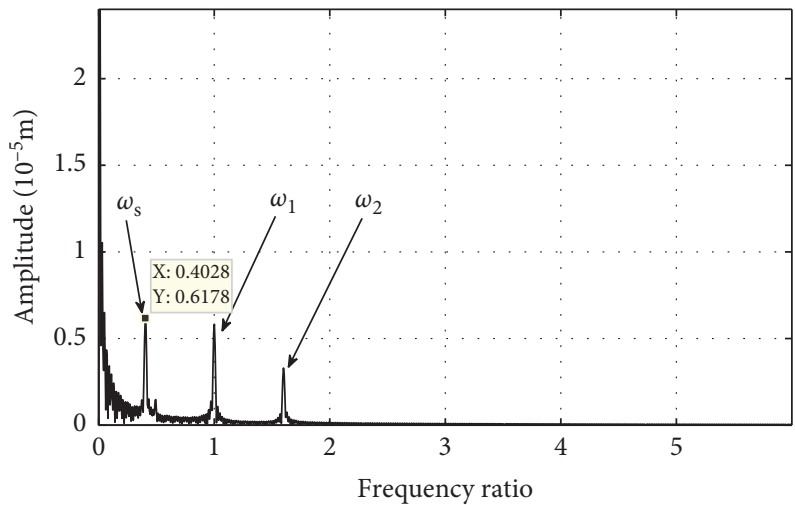

(e)

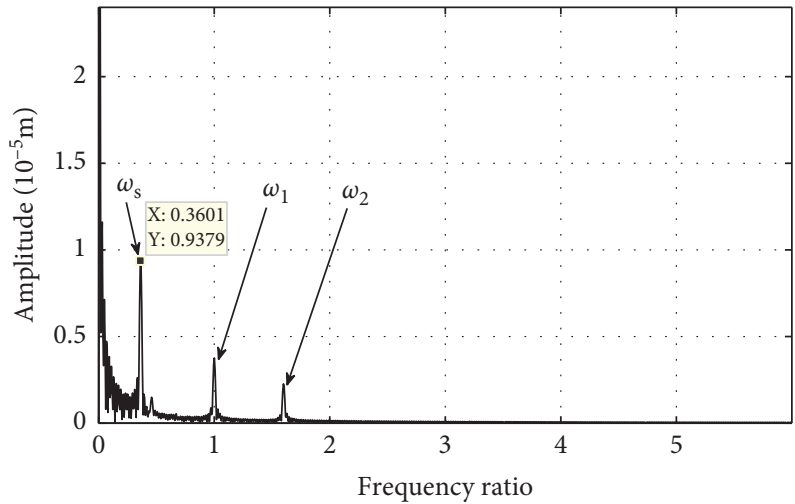

(g)

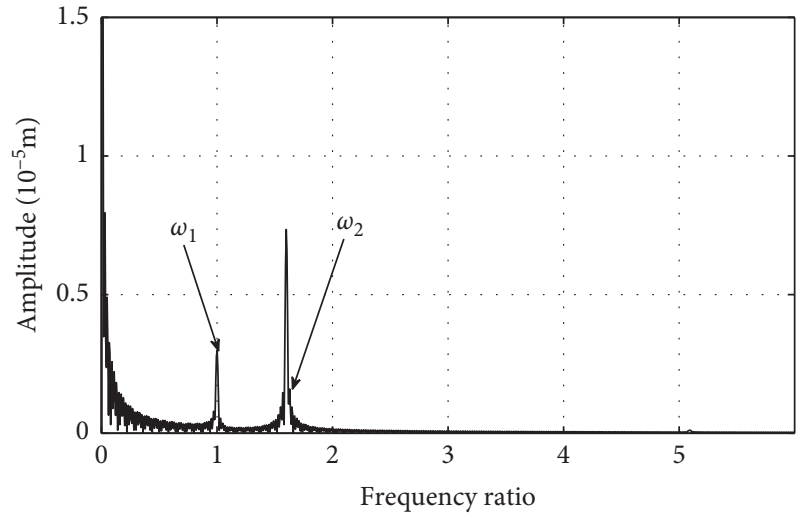

(b)

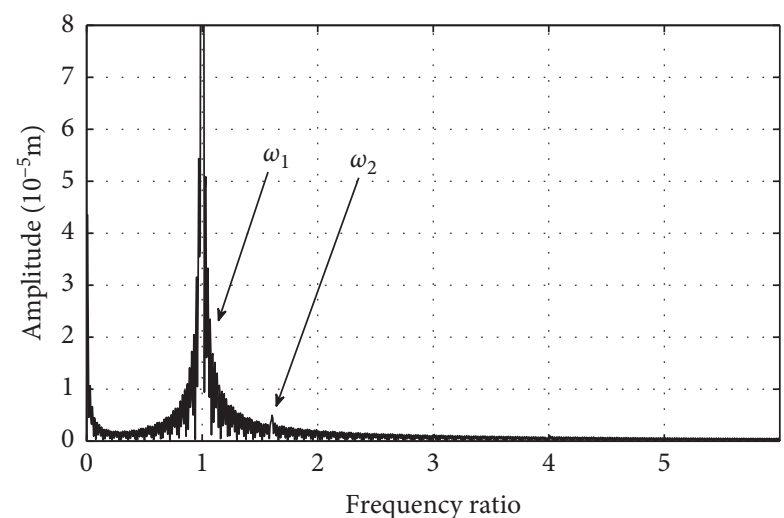

(d)

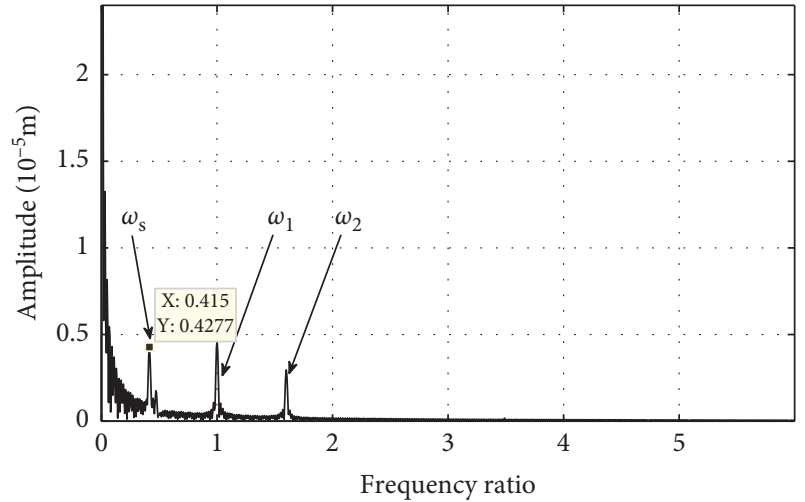

(f)

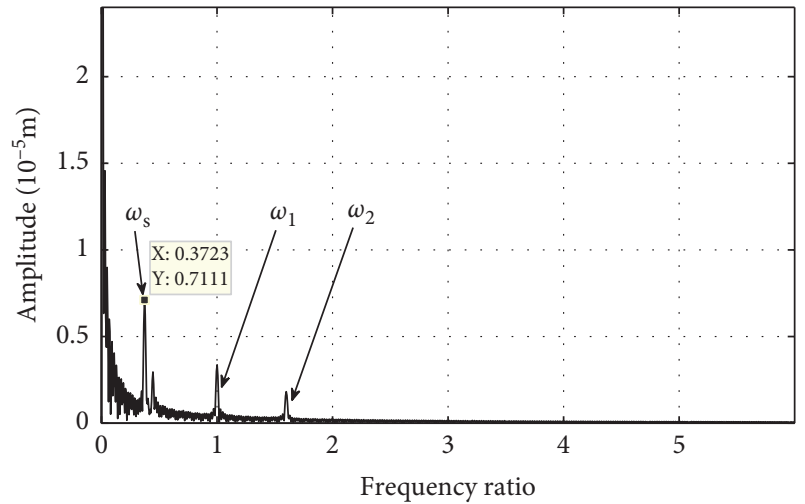

(h)

Figure 22: Spectrograms for the vertical vibration of disk 4. (a) $300 \mathrm{rad} / \mathrm{s}, \lambda=1.6$. (b) $300 \mathrm{rad} / \mathrm{s}, \lambda=-1.6$. (c) $400 \mathrm{rad} / \mathrm{s}, \lambda=1.6$. (d) $400 \mathrm{rad} / \mathrm{s}$, $\lambda=-1.6$. (e) $500 \mathrm{rad} / \mathrm{s}, \lambda=1.6$. (f) $500 \mathrm{rad} / \mathrm{s}, \lambda=-1.6$. (g) $550 \mathrm{rad} / \mathrm{s}, \lambda=1.6$. (h) $550 \mathrm{rad} / \mathrm{s}, \lambda=-1.6$. 


$$
\begin{aligned}
\dot{\psi} & =\frac{y \dot{x}-x \dot{y}}{x^{2}+y^{2}}, \\
\tan \psi & =\frac{x}{y},
\end{aligned}
$$

where $x$ and $y$ denote the eccentric position of the journal in the Cartesian coordinate system. $R, L$, and $c$ are the radius, length, and radial clearance of SFD, respectively, and $\mu$ denotes the dynamic viscosity. $I_{j}(j=1-3)$ are Sommerfeld integrals.

Similar to the nonlinear forces of rolling ball bearing as equations (13a)-(13e), the nonlinear forces of intermediate bearing can be represented as equations (15a) and (15b).

$$
\left\{\begin{array}{l}
f_{x}^{b}=-k_{n} \sum_{j=1}^{N_{b}} u_{\theta j}^{\xi} H\left(u_{\theta j}\right) \cos \theta_{j}, \\
f_{y}^{b}=-k_{n} \sum_{j=1}^{N_{b}} u_{\theta j}^{\xi} H\left(u_{\theta j}\right) \sin \theta_{j}, \\
u_{\theta j}=\left(x^{i r}-x^{\text {or }}\right) \cos \theta_{j}+\left(y^{i r}-y^{\text {or }}\right) \sin \theta_{j}-\frac{\gamma}{2},
\end{array}\right.
$$

where the superscripts " $i r$ " and "or" represent the internal and external rings of the bearing, respectively.

4.2. Calculation Model. The intermediate bearing-SFD-rotor system is presented in Figure 12. The rotor system is composed of two shafts, rotating around the same axis $o z$, which are linked by an intermediate bearing. There are two disks in each rotor, disks 1 and 4 in the inner rotor, and disks 2 and 3 in the outer rotor. The rotors are supported by four supports, and the supporting form of supports I, II, and III is elastic support + rolling ball bearing + SFD. Support IV is the intermediate bearing with internal ring connected with the inner rotor and external ring linked with the outer rotor. Inertia properties and unbalance configuration of the disks are shown in Table 5. Table 6 shows the radial stiffness of elastic supports. And the parameters of SFDs and intermediate bearing are shown in Tables 7 and 8 . The material density of the shafts is $7810 \mathrm{~kg} / \mathrm{m}^{3}$, Young's modulus is $196 \mathrm{GPa}$, and the shear modulus is $75.5 \mathrm{GPa}$. The speed ratio of the rotor system is defined as $\lambda=\omega_{2} / \omega_{1}$, where $\omega_{1}$ and $\omega_{2}$ are the rotation speeds of the inner and outer rotors, respectively, and $\lambda=1.6$ in this work.

4.3. Response Analysis. The finite element model of the dual rotor system is established, and the geometric dimensions and information of each element are listed in Table 9.

Taking hovering as the research object, the dynamic behavior of the complex intermediate bearing-SFD-rotor system under constant maneuvering overload is analyzed. The maneuvering parameters of the aircraft are shown in Table 10. Except for the maneuvering parameters mentioned here, the values of other parameters are set to 0 .
Solving the dynamic differential equations under the conditions of straight flight and maneuver flight, respectively, Figures 13 and 14 show the waterfall spectra plots for the vibration of disks 3 and 4 . And the spectrograms for the vertical vibration of disk 4 are shown in Figure 15. For the condition of straight flight, the frequency components mainly include the fundamental frequencies $\omega_{1}$ and $\omega_{2}$ and some combined frequency components such as $2 \omega_{2}-\omega_{1}$, $3 \omega_{2}-2 \omega_{1}$, caused by the coupling effect of the inner and outer rotors through the intermediate bearing, and the subharmonic vibration induced by the nonlinear characteristics of SFDs, which is defined as $\omega_{s}$. Compared with the straight flight condition, the combined frequency components are reduced, and the subharmonic vibration component is amplified under the maneuvering flight of hovering; see Figures 13(d), 14(d), and 15. It can be observed that the amplitude of the subharmonic component is relatively small when the rotating speed of the inner rotor is below $400 \mathrm{rad} / \mathrm{s}$, the $3 \mathrm{rd}$ critical speed of the rotor system. However, after the rotating speed exceeds $400 \mathrm{rad} / \mathrm{s}$, the subharmonic vibration is rapidly amplified with the continuous increase of the rotational speed, and it will far exceed the amplitude of fundamental frequency vibration when the rotating speed of the inner rotor reaches $550 \mathrm{rad} / \mathrm{s}$, as shown in Figure 15(h). Besides, similar to the bearing-rotor system, static displacements of the rotor can be observed under constant maneuvering overload. For $10 \mathrm{~g}$ overload in this work, the magnitude of the static displacement of the rotor in the horizontal direction caused by the additional centrifugal force has exceeded the resonance amplitude at the 3 rd critical speed of the rotor system. Therefore, for the future aircraft with high maneuverability and overload, the clearance of rotors and stators needs to be specially designed to prevent rub-impact fault.

The bifurcation diagrams of the vibration response varying with the rotational speed of disk 4 are shown in Figure 16. The orbits and Poincare maps for the vibration response of disk 4 are illustrated in Figures 17 and 18, respectively. By comparison, there is little difference in periodicity between the two conditions of straight flight and hovering at low speeds. However, as the rotating speed of the rotor increases, the vibration response periodicity will deteriorate under the condition of maneuver flight. Significantly, after the rotating speed of the inner rotor exceeds the 3rd critical speed of the rotor system, the movement of the rotor system gradually develops from pseudo-period to chaos under constant maneuvering overload.

The coaxial co- or counterrotating rotors exhibit different vibration characteristics [21, 23]. Under the condition of maneuver flight of hovering, the vibration characteristics of the dual rotor system in the case of counterrotation of the inner and outer rotors $(\lambda=-1.6)$ are analyzed in this work. In comparison with the two points, the waterfall spectra plots for the vibration of disks 3 and 4 are shown in Figures 19 and 20, respectively. The comparison results manifest that there are some differences in the static displacements between the two points under constant maneuvering overload. It is because the additional gyro torque acting on the rotor caused by maneuver flight is different in 
the two situations. And the magnitude of the static displacements relates to the specific structure of the rotor system, such as the axial locations of disks and bearings, the inertia properties of each disk, and the stiffness of each bearing.

The spectrograms for the vertical vibration of disks 3 and 4 are shown in Figures 21 and 22, respectively. Compared with the corotating dual rotor systems, the amplitude of subharmonic can be reduced in varying degrees under constant maneuvering overload in the case of counterrotation, as highlighted in Figures 21(e)-21(h) and 22(e)22(h). Therefore, the design of counterrotation of the inner and outer rotors can effectively reduce the amplitude of subharmonic induced by large maneuvering overload.

\section{Conclusions}

A nonlinear dynamic model of the bearing-rotor system under arbitrary maneuver flight conditions is modeled in this paper, and the added damping, stiffness, and maneuvering load generated by maneuver flight are obtained. The numerical integral method is used to investigate the vibration behavior of the rotor under constant maneuvering overload. Moreover, the simulation results are compared and verified by the experimental results. Based on this, the vibration characteristics of a complex intermediate bearingSFD-rotor system under constant maneuvering overload are analyzed. Conclusions are as follows:

(1) The maneuver flight action can lead to some static displacements of the rotor. It is found that the static displacements in the horizontal direction caused by the additional centrifugal force do not change with the rotating speed of the rotor. And the static displacements in the vertical direction induced by the additional gyro moment increase with the rise in the rotating speed. For the future aircraft with high maneuverability and overload, the clearance of rotors and stators needs to be specially designed to prevent rub-impact fault induced by the static displacements.

(2) Compared with the condition of straight flight, the combined frequency components induced by the coupling of the inner and outer rotors through the intermediate bearing are weakened. In contrast, the subharmonic vibration component caused by the nonlinear characteristics of SFDs is amplified under constant maneuvering overload. The amplitude of subharmonic vibration may far exceed the fundamental frequency vibration under certain maneuver flight conditions, which may do great harm to the bearings of rotor system, and it needs to be paid great attention in the design of aeroengine.

(3) The periodicity of motion of the rotor deteriorates during maneuver flight. For the dual rotor system under the maneuver action of hovering studied in this paper, when the rotating speed exceeds the $3 \mathrm{rd}$ critical speed of the rotor system, the movement of the rotor system develops from pseudo-period to chaos gradually as the rotational speed continues to increase.

(4) The co- and counterrotating dual rotor systems have different vibration characteristics under constant maneuvering overload. The design of counterrotation of the inner and outer rotors can effectively reduce the amplitude of subharmonic induced by large maneuvering overload.

\section{Data Availability}

The data that support the findings of this study are available upon request from the corresponding author.

\section{Conflicts of Interest}

The authors declare that there are no conflicts of interest regarding the publication of this paper.

\section{Acknowledgments}

This work was supported by the National Natural Science Foundation of China (grant number 51775266) and the National Science and Technology Major Project (grant number 2017-IV-0008-0045).

\section{References}

[1] Y. S. Chen and H. B. Zhang, "Review and prospect on research of dynamics of complete aero-engine system," Acta Aeronauticaet Astrnautica Sinica, vol. 32, no. 8, pp. 1371-1391, 2011, Chinese.

[2] H. N. Chen, K. S. Brentner, L. V. Lopes, and J. F. Horn, "A study of rotorcraft noise prediction in maneuvering flight," in Proceedings of the 42nd AIAA Aerospace Sciences Meeting and Exhibit, Reno, NV, USA, January 2004.

[3] M. Xu and M. F. Liao, "The vibration performance of the jeffcott rotor system with sfd in maneuver flight," Journal of Aerospace Power, vol. 18, no. 3, pp. 394-401, 2003, Chinese.

[4] S. Ananthan and J. G. Leishman, "Helicopter rotor wake dynamics during simulated tactical maneuvers," in Annual Forum Proceedings-American Helicopter Society, Baltimore, MD, USA, June 2004.

[5] M. Sakata, K. Kimura, S. K. Park, and H. Ohnabe, "Vibration of bladed flexible rotor due to gyroscopic moment," Journal of Sound and Vibration, vol. 131, no. 3, pp. 417-430, 1989.

[6] M. Sakata, K. Kimura, S. Okamoto, and K. Oikawa, "Vibration analysis of a high speed and light weight rotor system subjected to a pitching or turning motion," Journal of Sound and Vibration, vol. 184, no. 5, pp. 871-885, 1995.

[7] H.-N. Chen, K. S. Brentner, L. V. Lopes, and J. F. Horn, “An initial analysis of transient noise in rotorcraft maneuvering flight," International Journal of Aeroacoustics, vol. 5, no. 2, pp. 109-138, 2006.

[8] G. A. Brès, K. S. Brentner, G. Perez, and H. E. Jones, "Maneuvering rotorcraft noise prediction," Journal of Sound and Vibration, vol. 275, no. 3-5, pp. 719-738, 2004.

[9] Y. Yang, X. Ren, W. Qin, Y. Wu, and X. Zhi, "Analysis on the nonlinear response of cracked rotor in hover flight," Nonlinear Dynamics, vol. 61, no. 1-2, pp. 183-192, 2010. 
[10] F. S. Lin and G. Meng, "Study on the dynamics of a rotor in a maneuvering aircraft," Journal of Vibration and Acoustics, vol. 125, no. 3, pp. 1220-1227, 2003.

[11] F. S. Lin and G. Meng, "Study on the dynamic characteristics of SFD-rotor system in aircrafts," Journal of Vibration Engineering, vol. 17, no. 4, pp. 403-408, 2004, Chinese.

[12] C. S. Zhu and Y. J. Chen, "Vibration characteristics of aeroengine's rotor system during maneuvering flight," Acta Aeronautica Et Astronautica Sinica, vol. 27, no. 5, pp. 835841, 2006, Chinese.

[13] C. S. Zhu and Y. J. Chen, "General dynamic model of aeroengine's rotor system during maneuvering flight," Journal of Aerospace Power, vol. 24, no. 2, pp. 371-377, 2009, Chinese.

[14] L. Hou and Y. Chen, "Analysis of $1 / 2$ sub-harmonic resonance in a maneuvering rotor system," Science China Technological Sciences, vol. 57, no. 1, pp. 203-209, 2014.

[15] L. Hou, Y. Chen, Q. Cao, and Z. Zhang, “Turning maneuver caused response in an aircraft rotor-ball bearing system," Nonlinear Dynamics, vol. 79, no. 1, pp. 229-240, 2015.

[16] L. Hou, Y. Chen, Q. Cao, and Z. Lu, "Nonlinear vibration analysis of a cracked rotor-ball bearing system during flight maneuvers," Mechanism and Machine Theory, vol. 105, pp. 515-528, 2016.

[17] T. Gao, S. Cao, and Y. Sun, "Nonlinear dynamic behavior of a flexible asymmetric aero-engine rotor system in maneuvering flight," Chinese Journal of Aeronautics, vol. 33, no. 10, pp. 2633-2648, 2020.

[18] B. Han and Q. Ding, "Forced responses analysis of a rotor system with squeeze film damper during flight maneuvers using finite element method," Mechanism and Machine Theory, vol. 122, pp. 233-251, 2018.

[19] M. I. Friswell, J. E. T. Penny, S. D. Garvey, and A. W. Lees, Dynamics of Rotating Machines, Cambridge University Press, Cambridge, UK, 2010.

[20] G. Chen and R. T. Hou, "Nonlinear dynamic response analysis of unbalance rubbing coupling faults of rotor ball bearing stator coupling system," Journal of Aerospace Power, vol. 22, no. 10, pp. 1771-1778, 2007, Chinese.

[21] X. G. Yang, G. H. Luo, Z. H. Tang, and F. Wang, "Modeling method and dynamic characteristics of high-dimensional counter-rotating dual rotor system," Journal of Aerospace Power, vol. 29, no. 3, pp. 585-595, 2014, Chinese.

[22] H.-L. Zhou, G.-H. Luo, G. Chen, and F. Wang, "Analysis of the nonlinear dynamic response of a rotor supported on ball bearings with floating-ring squeeze film dampers," Mechanism and Machine Theory, vol. 59, pp. 65-77, 2013.

[23] F. Wang, G.-H. Luo, S. Yan, and H.-T. Cui, "A comparison study on Co- and counterrotating dual-rotor system with squeeze film dampers and intermediate bearing," Shock and Vibration, vol. 2017, no. 4, pp. 1-25, Article ID 5493763, 2017. 